\title{
Effect of realistic nuclear charge distributions on isotope shifts and progress towards the extraction of higher-order nuclear radial moments
}

\author{
A. Papoulia, ${ }^{1}$ B. G. Carlsson,,${ }^{1, *}$ and J. Ekman ${ }^{2}$ \\ ${ }^{1}$ Division of Mathematical Physics, LTH, Lund University, Post Office Box 118, S-22100 Lund, Sweden \\ ${ }^{2}$ Group for Materials Science and Applied Mathematics, Malmö University, S-20506 Malmö, Sweden
}

(Received 13 April 2016; published 3 October 2016)

\begin{abstract}
Atomic spectral lines from different isotopes display a small shift in energy, commonly referred to as the line isotope shift. One of the components of the isotope shift is the field shift, which depends on the extent and the shape of the nuclear charge density distribution. The purpose of this work is to investigate how sensitive field shifts are with respect to variations in the nuclear size and shape and what information of nuclear charge distributions can be extracted from measurements. Nuclear properties are obtained from nuclear density functional theory calculations based on the Skyrme-Hartree-Fock-Bogoliubov approach. These results are combined with multiconfiguration Dirac-Hartree-Fock methods to obtain realistic field shifts and it is seen that phenomena such as nuclear deformation and variations in the diffuseness of nuclear charge distributions give measurable contributions to the isotope shifts. Using a different approach, we demonstrate the possibility to extract information concerning the nuclear charge densities from the observed field shifts. We deduce that combining methods used in atomic and nuclear structure theory gives an improved description of field shifts and that extracting additional nuclear information from measured isotope shifts is possible in the near future with improved experimental methods.
\end{abstract}

DOI: 10.1103/PhysRevA.94.042502

\section{INTRODUCTION}

Information of nuclear sizes has grown rapidly during the last decades. In the compilation by Angeli and Marinova in 2013 [1], root-mean-square (rms) radii were reported for more than 900 isotopes of which the majority are radioactive systems. This development is a consequence of refined experimental and theoretical methods, and a state-of-the-art example is the frequency comb measurement of the hydrogendeuterium radius difference by Parthey et al. [2]. The plenitude of available data has allowed for detailed investigations of the evolution of nuclear radii for isotope sequences along virtually the entire periodic table. These studies have revealed unexpected trends, especially close to magic numbers, which serve as benchmarks for nuclear structure calculations [3].

However, more detailed and model-independent experimental information of nuclear charge distributions beyond the rms radius is only available for stable or long-lived isotopes from electron scattering experiments. On the theoretical side it has been shown that isotope shifts in heavier systems depend on the nuclear model used [4] and that the contribution from nuclear deformation to the isotope shift in some cases is comparable to the uncertainty in recent dielectronic recombination experiments $[5,6]$.

Experimental techniques such as high-precision laser measurements at the COLLAPS and CRIS experiments at ISOLDE/CERN [7] and dielectronic recombination experiments at the envisaged realization of CRYRING at GSI [8] are constantly evolving. This justifies a more systematic theoretical investigation of what information can be revealed about nuclear charge distributions in exotic systems.

The main objective of this work is to study the effect of realistic charge distributions, taken from nuclear density

\footnotetext{
*gillis.carlsson@matfys.lth.se
}

functional theory (DFT), on the isotope shift in heavier atoms. In addition, a promising method for the extraction of higher-order radial moments from experimental isotope shifts is also presented and tested.

\section{ISOTOPE SHIFTS}

The atomic nucleus is $\sim 10^{4}$ smaller than the size of the atom. Even so, the finite mass and extended charge distribution of the nucleus have a measurable effect on atomic spectra. Spectral lines from different isotopes display a small shift in energy referred to as the isotope shift (IS), which can further be decomposed into a mass shift (MS) and a field shift (FS) contribution. The difference in energy between the corresponding atomic level $i$ of two isotopes $A$ and $A^{\prime}$, the level isotope shift, can thus be expressed as

$$
\delta E_{i, \mathrm{IS}}^{A, A^{\prime}}=\delta E_{i, \mathrm{MS}}^{A, A^{\prime}}+\delta E_{i, \mathrm{FS}}^{A, A^{\prime}}=E_{i}^{A^{\prime}}-E_{i}^{A} .
$$

For a particular atomic transition $k$ between upper $u$ and lower $l$ levels, the difference in energy for a pair of isotopes, namely, the line frequency isotope shift, is consequently given by

$$
\begin{aligned}
\delta v_{k, \mathrm{IS}}^{A, A^{\prime}} & =\delta v_{k, \mathrm{MS}}^{A, A^{\prime}}+\delta v_{k, \mathrm{FS}}^{A, A^{\prime}}=v_{k}^{A^{\prime}}-v_{k}^{A} \\
& =\frac{\delta E_{u, \mathrm{IS}}^{A, A^{\prime}}-\delta E_{l, \mathrm{IS}}^{A, A^{\prime}}}{h} .
\end{aligned}
$$

The level mass shift contribution can be expressed as

$$
\delta E_{i, \mathrm{MS}}^{A, A^{\prime}}=\left(\frac{M^{\prime}-M}{M M^{\prime}}\right) K_{\mathrm{MS}}^{i},
$$

where $M$ and $M^{\prime}$ are the atomic masses of the isotopes and $K_{\mathrm{MS}}^{i}$ is the mass-independent mass shift parameter [9-11]. Although the computation of the mass shift parameters, and hence the mass shift contribution to the isotope shift, represents a challenging task, it is not the main focus of this work. Instead, 
the focus here is on the extent and shape of nuclear charge distributions which almost exclusively affect the field shift described in detail below.

\section{A. Field shift}

The field shift arises from differences in the nuclear charge density distribution between isotopes caused by the different number of neutrons. Unlike pointlike charge distributions, more realistic charge distributions alter the central field that the atomic electrons experience, and hence the atomic level and transition energies will be affected. Evidently, the field shift effect is more pronounced for electrons moving in $s_{1 / 2}$ and $p_{1 / 2}$ orbitals due to the nonzero probability of the radial wave functions at the origin. Moreover, the nuclear charge and extent, together with the contraction of the atomic orbitals, increase with the proton number $Z$ and thus the contribution from the field shift to the isotope shift is found to be dramatically larger in heavier systems.

\section{Nonperturbative "exact" method}

In atomic structure calculations, where the contribution from the mass shift is neglected, the level field shift can be computed according to Eq. (1) by performing separate calculations for two isotopes $A$ and $A^{\prime}$, with different parameter sets describing the respective nuclear charge distributions. This method is in general highly model dependent since the description of the nucleus is normally restricted to an approximate model. Moreover, this procedure is cumbersome if calculations are to be performed for many isotope pairs and in addition it may suffer from numerical instabilities since it involves the substraction of large quantities (atomic binding energies) to obtain a tiny quantity. Nevertheless, this strategy constitutes an "exact" method for estimating the validity of perturbative approaches and the resulting field shifts will be denoted $\delta v_{k, \mathrm{VA}}^{\text {exact }}$ below.

\section{Perturbative method}

To eliminate the disadvantages of the exact method described above and allow for a more flexible analysis of the field shift, an alternative approach based on perturbation theory may be used. Within the framework of perturbation, the first-order level field shift of level $i$ can be written

$$
\delta E_{i, \mathrm{FS}}^{(1) A, A^{\prime}}=-\int_{R^{3}}\left[V_{A^{\prime}}(\boldsymbol{r})-V_{A}(\boldsymbol{r})\right] \rho_{i}^{e}(\boldsymbol{r}) d^{3} \boldsymbol{r},
$$

where $V_{A}(\boldsymbol{r})$ and $V_{A^{\prime}}(\boldsymbol{r})$ are the one-electron potentials arising from the different nuclear charge distributions of the two isotopes and $\rho_{i}^{e}(\boldsymbol{r})$ is the electron density inside the nuclear volume of the reference isotope $A$.

Following the work by Seltzer [12], Torbohm et al. [13], and Blundell et al. [14] and assuming an extended spherical symmetric nuclear charge distribution, it can be shown that the electron density to a very good approximation can be expanded around $r=0$ as an even polynomial function keeping only the first few terms:

$$
\rho_{i}^{e}(\boldsymbol{r}) \approx b_{i}(r)=b_{i, 1}+b_{i, 2} r^{2}+b_{i, 3} r^{4}+b_{i, 4} r^{6} .
$$

Inserting the expression above in Eq. (4) and making use of the Laplacian operator in spherical coordinates, $\nabla^{2} r^{2 N}=2 N(2 N+1) r^{2 N-2}$, Poisson's equation $\nabla^{2} V_{A}(\boldsymbol{r})=$ $-4 \pi \rho_{A}(\boldsymbol{r})$, and finally Eq. (2), the first-order line frequency field shift is given by $[14,15]$

$$
\delta v_{k, \mathrm{FS}}^{(1) A, A^{\prime}} \approx \delta v_{k, \mathrm{RFS}}^{A, A^{\prime}}=\sum_{N=1}^{4} F_{k, N} \delta\left\langle r^{2 N}\right\rangle^{A, A^{\prime}},
$$

where $F_{k, N}$ are the so-called line electronic factors expressed as

$$
F_{k, N}=\frac{2 \pi}{h} \frac{Z \Delta b_{k, N}}{N(2 N+1)}
$$

and

$$
\delta\left\langle r^{2 N}\right\rangle^{A, A^{\prime}}=\left\langle r^{2 N}\right\rangle^{A}-\left\langle r^{2 N}\right\rangle^{A^{\prime}}
$$

are the differences of the nuclear radial moments, of order $2 N$, of the isotopes $A$ and $A^{\prime}$. The electronic factors are proportional to the difference of the electronic density inside the nucleus between the upper and lower atomic level, thus, $\Delta b_{k, N}=b_{u, N}-b_{l, N}$.

The reformulated field shift (RFS) according to Eq. (6) enables a more versatile analysis of field shifts. This is due to the fact that the radial moments $\left\langle r^{2 N}\right\rangle$ used in the expression can be taken from any model, calculation, or experiment. In addition, it is possible to analyze the contributions to the field shift order by order. For example, keeping only the first term in Eq. (6) we obtain

$$
\delta \nu_{i, \mathrm{FS}}^{(1) A, A^{\prime}} \approx \frac{2 \pi}{3 h} Z \Delta \rho_{i}^{e}(\mathbf{0}) \delta\left\langle r^{2}\right\rangle^{A, A^{\prime}},
$$

which is a suitable approximation for lighter systems where a constant electron density within the nucleus can be assumed, $\rho_{i}^{e}(\boldsymbol{r}) \approx b_{i, 1}=\rho_{i}^{e}(\mathbf{0})$. For heavier systems, however, the electron density varies inside the nuclear volume and thus the $N \geqslant 2$ terms in Eq. (6) must also be considered for an accurate description. Further on, by including these higher-order contributions, the effect on the isotope shift due to details in the nuclear charge distribution can be analyzed. As we shall see, the reversed approach is also possible, namely, to extract higher-order radial moments of the nuclear charge distribution from observed isotope shifts.

\section{B. Computational procedure}

Solutions to the many-body Hamiltonian describing the atom are obtained by performing calculations using the relativistic atomic structure package GRASP2K [16], which is based on the multiconfiguration Dirac-Hartree-Fock (MCDHF) approach. In the MCDHF method, atomic state functions $\Psi\left(\gamma P J M_{J}\right)$, which are approximate solutions to the DiracCoulomb Hamiltonian, are expanded over configuration state functions (CSFs), $\Phi\left(\gamma_{i} P J M_{J}\right)$, with appropriate total angular momentum $(J)$ symmetry and parity $P$ :

$$
\Psi\left(\gamma P J M_{J}\right)=\sum_{i=1}^{N} c_{i} \Phi\left(\gamma_{i} P J M_{J}\right) .
$$

In the expression above, $\gamma_{i}$ represents the configuration, coupling, and other quantum number necessary to uniquely describe the state $i, M_{J}$ is the projection of $J$ on the $z$ axis, and $c_{i}$ are mixing coefficients fulfilling the condition $\sum_{i=1}^{N} c_{i}^{2}=1$. 
The CSFs are constructed from one-electron Dirac orbitals that together with the mixing coefficents are obtained in a relativistic self-consistent-field procedure by applying the variational principle [17]. The transverse photon interaction as well as leading quantum electrodynamic (QED) corrections can be accounted for in subsequent relativistic configuration interaction (RCI) calculations [18].

Once a set of ASFs is obtained, the computation of the isotope shift parameters is carried out using the program RIS4 [15], which represents an extension of the predecessor RIS3 [19]. In RIS4 the polynomial expansion $b_{i}(r)$ given by Eq. (5) is for each level fitted to the constructed electron density $\rho_{i}^{e}(\boldsymbol{r})$ using a least-squares method. Finally, by combining the expansion coefficients $b_{i, N}$ from two or more levels, the line electronic factors are computed for the reference isotope $A$ according to Eq. (7).

\section{REALISTIC NUCLEAR CHARGE DISTRIBUTIONS}

As seen above, the reformulated field shift depends on the radial moments of the nuclear charge distribution. These moments can be calculated from nuclear models that provide accurate charge distributions. In this section, three such models are compared.

\section{A. Nuclear charge distribution models}

The nuclear charge distribution can be approximated by an analytical expression such as the Fermi distribution

$$
\rho(r, \theta)=\frac{\rho_{0}}{1+e^{\frac{r-c(\theta)}{a}}},
$$

where, if only axially symmetric quadrupole deformation is considered, $c(\theta)=c_{0}\left[1+\beta_{20} Y_{20}(\theta)\right]$. This modified Fermi distribution has been used previously to investigate the effect of deformation on atomic binding energies in Li-like systems [5,20,21]. In these studies, nuclear deformation parameters extracted from highly accurate muonic atom studies were used [22,23]. The value of $\rho_{0} \approx \rho(r=0)$ is determined by the normalization condition

$$
\int \rho(\boldsymbol{r}) d \boldsymbol{r}=1
$$

and the parameter $\alpha$ is given by the relation

$$
t=4 \ln (3) \alpha,
$$

where $t$ is the skin thickness of the distribution. The skin thickness is defined as the interval where the density decreases from $90 \%$ to $10 \%$ of $\rho(0)$. The parameter $c_{0}$ reflects the size of the nucleus.

In the GRASP2K code [16], the explicit values for these parameters are taken as [4] $t=2.3 \mathrm{fm}, \beta_{20}=0$ and the parameter $c_{0}$ is chosen so that the rms radius of the nuclear charge distribution becomes

$$
\sqrt{\left\langle r^{2}\right\rangle}=0.836 A^{\frac{1}{3}}+0.570 \mathrm{fm}(A>9),
$$

where $A$ denotes the number of nucleons of the isotope.

Realistic nuclear charge distributions can also be obtained from microscopic nuclear models based on effective interactions. Such models have the advantage that the size, shape, and diffuseness of the nuclear density is obtained by solving a selfconsistent set of Hartree-Fock-Bogoliubov (HFB) equations.

In this work, we adopt the effective Skyrme interaction [24] and consider two different sets of Skyrme parameters called SLY4 and UNEDF1. The parameters in both sets are adjusted to fit experimental data in a broad range of nuclei. The SLY4 set was fitted with an emphasis on describing neutron-rich nuclei [25], whereas the UNEDF1 set constitutes a more recent parametrization fitted to reproduce both ground-state energies as well as radii and single-particle energies [26]. In spherical symmetry, the solutions to the HFB equations are provided by the code HOSPHE (v2.00), which is a new version of the program HOSPHE (v1.02) [27]. In the case of deformed nuclei, we use the code HFBTHO (2.00d) [28], based on a cylindrically deformed harmonic oscillator (HO) basis.

For spherical nuclei, we take into account the finite nature of protons by folding the densities using the convolution formula

$$
\varrho_{c}(\boldsymbol{r})=\int d^{3} \boldsymbol{r}^{\prime} \rho_{p}\left(\boldsymbol{r}^{\prime}\right) g\left(\left|\boldsymbol{r}-\boldsymbol{r}^{\prime}\right|\right),
$$

where $\rho_{p}(\boldsymbol{r})$ is the initially calculated proton density and

$$
g(\boldsymbol{r})=\left(r_{0} \sqrt{\pi}\right)^{-3} e^{-\left(\boldsymbol{r} / r_{0}\right)^{2}}
$$

the proton form factor, assumed to be a Gaussian with $r_{0}=$ $\sqrt{\frac{2}{3}} r_{p}^{\mathrm{rms}}$, where $r_{p}^{\mathrm{rms}}$ is the proton rms radius [29]. Experiments to determine the proton radius have resulted in different values of $r_{p}^{\text {rms }}[30,31]$, and in this work we adopt the results based on electron scattering measurements assuming $r_{p}^{\mathrm{rms}}=0.88 \mathrm{fm}$.

In Fig. 1, the theoretical $\mathrm{rms}$ radii are compared to experimental data obtained from elastic electron scattering experiments [32,33]. A total of 16 spherical isotopes of various elements, $\mathrm{O}, \mathrm{S}, \mathrm{Ca}, \mathrm{Ni}, \mathrm{Sn}$, and $\mathrm{Pb}$, are used in the comparison. As seen in this figure, both the nuclear models as well as the empirical parametrization [Eq. (14)] are in good agreement with the experimental data.

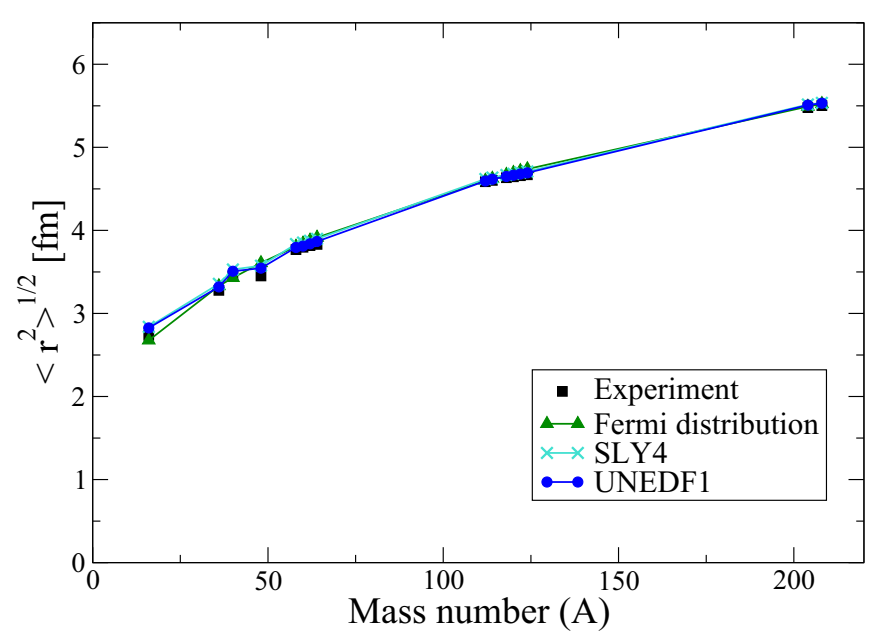

FIG. 1. Rms radii of theoretical charge distributions compared to experimental data. Two different Skyrme parameter sets, SLY4 and UNEDF1, are used with moments calculated after taking into account the finite proton size. The resulting $\sqrt{\left\langle r^{2}\right\rangle}$ values from the Fermi distribution used in the GRASP2K code [Eq. (14)] are also included. 

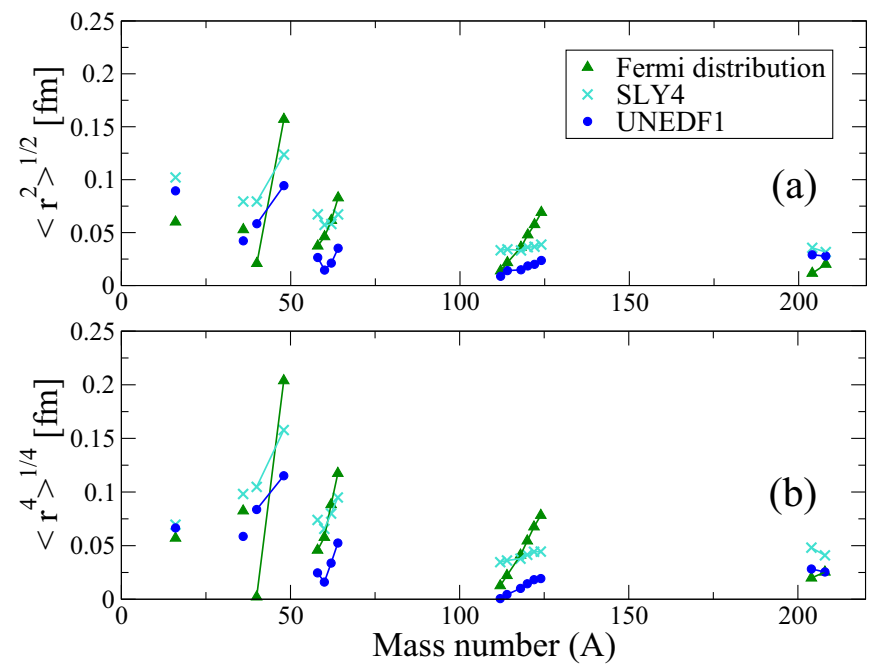

FIG. 2. Discrepancy of (a) the rms radii $\sqrt{\left\langle r^{2}\right\rangle}$ and (b) the $\sqrt[4]{\left\langle r^{4}\right\rangle}$ moment of the theoretical charge distributions compared to experimental data. Isotopic sequences are connected with lines.

The discrepancy between the theoretical and the experimental $\sqrt{\left\langle r^{2}\right\rangle}$ values is shown in Fig. 2(a). As seen in this figure, the more recent Skyrme parameters (UNEDF1) give the best description of the data. The two microscopic models also stand out as they are in general better at capturing the isotopic trends giving flatter curves than the Fermi distribution.

For calculations of field shifts, the higher-order moments may also play an important role and in Fig. 2(b) the discrepancy in the prediction of the $\sqrt[4]{\left\langle r^{4}\right\rangle}$ values is shown. This comparison shows the same trend as for the $\sqrt{\left\langle r^{2}\right\rangle}$ values, namely, that microscopic models capture the isotopic trends better while the Fermi distribution in general does a good job for the stable nuclei. One might consider using more refined empirical expressions containing a dependence on the difference in proton and neutron numbers, but since such an approach would anyway not capture the important changes caused by deformations, the best approach comes from using state-ofthe-art microscopic nuclear models.

In Table I, the standard deviations of the discrepancies for the three models are compared. Considering the average agreement, the Fermi distribution and the Skyrme-SLY4 give similar results while the more recent UNEDF1 is significantly better. In addition, the UNEDF1 set predicts the $\sqrt{\left\langle r^{2}\right\rangle}$ and $\sqrt[4]{\left\langle r^{4}\right\rangle}$ moments with about the same precision, while the precision deteriorates slightly for the two other models. This agrees with the fact that the full density profiles also tend to be better reproduced by UNEDF1. Higher-order moments are

TABLE I. Standard deviations of discrepancies in $\sqrt{\left\langle r^{2}\right\rangle}$ and $\sqrt[4]{\left\langle r^{4}\right\rangle}$, calculated for the three theoretical models.

\begin{tabular}{lcc}
\hline \hline & $\sqrt{\left\langle r^{2}\right\rangle}$ & $\sqrt[4]{\left\langle r^{4}\right\rangle}$ \\
\hline Fermi distribution & 0.01660 & 0.01954 \\
Skyrme-SLY4 & 0.01821 & 0.01905 \\
Skyrme-UNEDF1 & 0.01271 & 0.01260 \\
\hline \hline
\end{tabular}

difficult to compare since more focus is then shifted towards the surface and tail of the density where insufficient precision in the data hampers a qualitative comparison. All in all, the UNEDF1 parametrization describes the nuclear charge distributions more accurately than both the Skyrme-SLY4 and Fermi distributions and therefore realistic nuclear radial moments resulting from this interaction will be used in the following in order to estimate the line field shifts.

\section{B. Application to line field shifts}

In this section, the atomic physics calculations for the electron energies are combined with the use of the microscopic nuclear models for the charge densities. As an example, we consider the resonance transition $6 s^{2}{ }^{1} S_{0} \longrightarrow 6 s 6 p^{1} P_{1}^{o}$ observed in several neutral $\mathrm{Ba}$ isotopes. By comparing the line field shift in the isotope series one may be able to draw conclusions on the shape and size of the nuclear density distributions. The most abundant barium isotope on Earth, ${ }^{138} \mathrm{Ba}$, is taken as a reference and the shifts in electron energies are thus compared to the values for this isotope. This reference isotope is spherical, while the other isotopes obtained by removing or adding a couple of neutrons are predicted to have more deformed shapes.

Figure 3 shows the calculated line field shifts for the $\mathrm{Ba}$ isotope series compared to experimental isotope shifts [34], where theoretical mass shift contributions have been subtracted [35]. The calculations based on the Fermi distribution show a linear dependence on the mass number $A^{\prime}$ of the target isotope and fail to capture the general trend. The microscopic nuclear calculations capture both the right trend with neutron number and in addition some of the odd-even staggering.

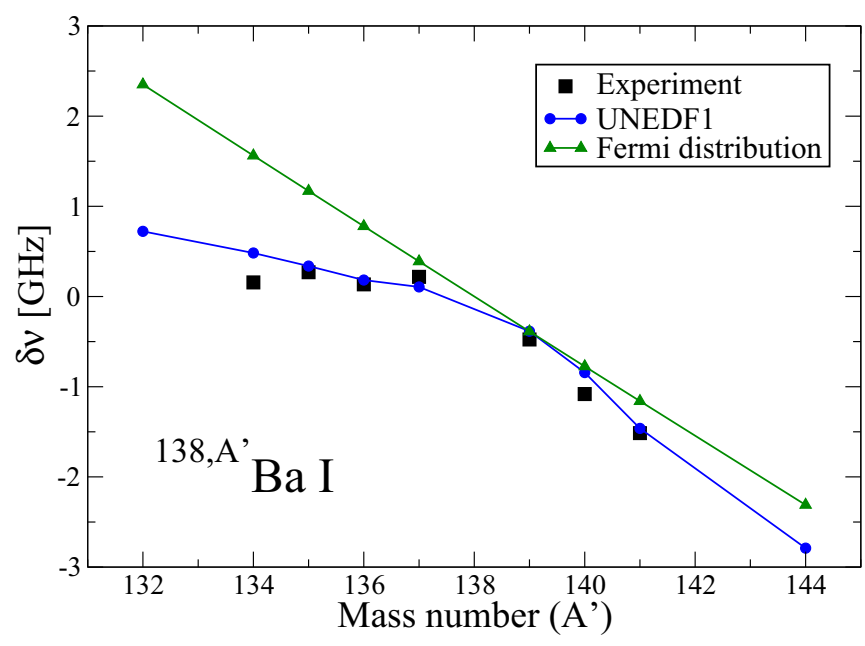

FIG. 3. The absolute line field shift values are compared to the available experimental data $[34,35]$. Nuclear radial moments resulted from the realistic HFB calculations using the Skyrme-UNEDF1 interaction, as well as from the Fermi distribution, have been used. All plotted values refer to the $6 s^{2}{ }^{1} S_{0} \longrightarrow 6 s 6 p{ }^{1} P_{1}^{o}$ transition. 


\section{EFFECT OF REALISTIC CHARGE DISTRIBUTIONS ON THE LINE FIELD SHIFTS}

In order to investigate the resulting field shifts when replacing the commonly adopted Fermi distribution with more realistic nuclear models, we examine the differences in the predicted field shifts for a variety of isotopes. For such analysis, the Fermi distribution is fitted so that it has the same $\left\langle r^{2}\right\rangle$ value as computed from the realistic distributions. Then,

$$
\begin{aligned}
\delta \nu_{\text {Fermi }}= & F_{k, 1} \delta\left\langle r^{2}\right\rangle_{\text {realistic }} \\
& +\sum_{N=2}^{4} F_{k, N} \delta\left\langle r^{2 N}\right\rangle_{\text {Fermi }} .
\end{aligned}
$$

Thus, the correction when using realistic charge distributions is given by

$$
\delta v_{\text {realistic }}-\delta v_{\text {Fermi }}=\sum_{n=2}^{4} F_{k, N}\left[\delta\left\langle r^{2 N}\right\rangle_{\text {realistic }}-\delta\left\langle r^{2 N}\right\rangle_{\text {Fermi }}\right]
$$

In the following two subsections, the size of this correction term will be investigated for lithium-like and neutral systems.

\section{A. Li-like systems}

Isotope shifts in lithium-like systems have been studied theoretically and experimentally in the past [5,6,36-38] and are thus of particular interest. In Fig. 4, the magnitude of the "correction term" $\delta v_{\text {realistic }}-\delta v_{\text {Fermi }}$ for one of the resonance transitions has been plotted as a function of the mass number $A^{\prime}$ of the target isotope for a wide range of Li-like systems. For the spherical $\mathrm{Sn}, \mathrm{Pb}, \mathrm{Er}$, and Lv nuclear systems the magnitude
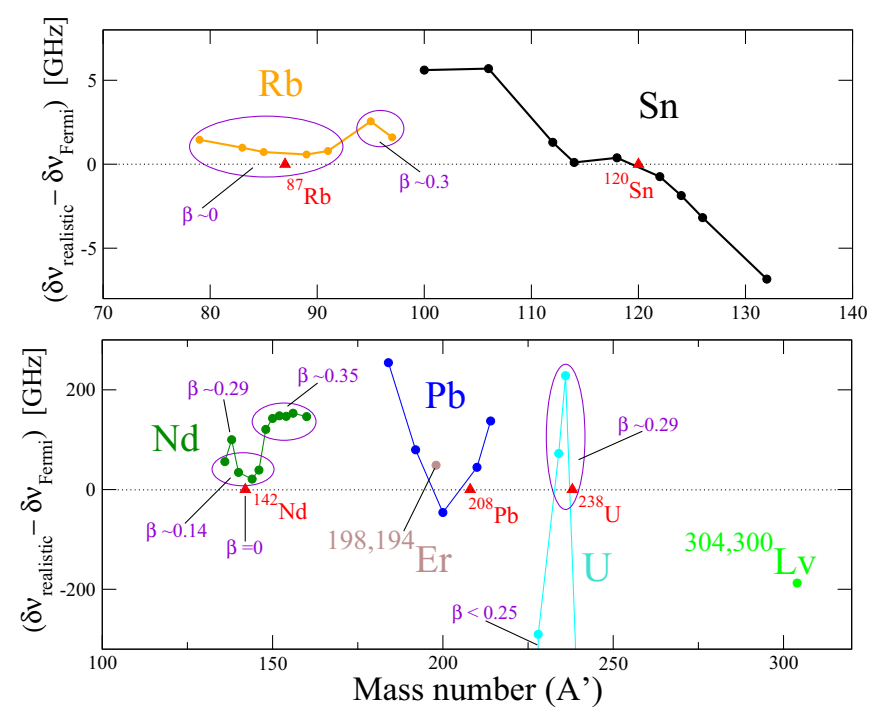

FIG. 4. The corrections $\delta v_{\text {realistic }}-\delta v_{\text {Fermi }}$ to the line field shift calculations as a function of the mass number $A^{\prime}$ of the target isotope for various Li-like systems. For the systems that contain deformed isotopes, the magnitude of the quadrupole deformation parameter $\beta_{20}$ of the target isotopes $A^{\prime}$ is indicatively shown. The isotopes used as reference are marked with triangles and all plotted values refer to the $1 s^{2} 2 s^{2} S_{1 / 2} \longrightarrow 1 s^{2} 2 p^{2} P_{1 / 2}^{o}$ resonance transition. of the corrections increases with $A^{\prime}$. Moreover, the absolute magnitude of the $\delta v_{\text {realistic }}-\delta v_{\text {Fermi }}$ term increases with the difference between the neutron number $\Delta N^{A, A^{\prime}}$ in the isotope sequences of $\mathrm{Sn}$ and $\mathrm{Pb}$. When more neutrons are added, they alter the protons distribution, leading to changes in the diffuseness. This effect is not included in the Fermi model where a constant skin thickness $t \simeq 2.3 \mathrm{fm}$ is assumed and may be a reason for the observed difference.

In the deformed $\mathrm{Rb}, \mathrm{Nd}$, and $\mathrm{U}$ systems, the corrections depend on the size of the nuclei as well as the quadrupole deformation parameter $\beta_{20}$, which is assumed to be zero in the spherical Fermi model. Hence, for large deformations the corrections for the $\mathrm{Rb}$ and $\mathrm{Nd}$ isotope pairs are comparable to the ones obtained for the spherical $\mathrm{Sn}$ and $\mathrm{Pb}$ isotope pairs. For the heavier $U$ isotopes, the corrections become significantly large in spite of the small difference in deformation between the reference and target isotopes.

In Fig. 5, the magnitude of the corrections has been plotted as a function of the calculated deformation parameter $\beta_{20}$ corresponding to the isotope $A^{\prime}$ for some $\mathrm{Nd}$ and $\mathrm{U}$ isotope pairs. In both plots, the magnitude of the "correction term" increases as the difference between the deformation of reference and target isotope becomes large. The largest corrections are obtained for the uranium isotope pairs ${ }^{240,238} \mathrm{U}$ and ${ }^{220,238} \mathrm{U}$. In this case, the correction amounts to $\sim 2.3 \%$ and $\sim 2 \%$, respectively.

The two-parameter Fermi model does not take into account the effect of deformation. As a result, the effect of realistic charge distributions on the field shifts is larger in atomic systems with deformed nuclei. The correction term $\delta v_{\text {realistic }}-\delta v_{\text {Fermi }}$ can, however, be decomposed into two parts and written as

$$
\begin{aligned}
\delta v_{\text {realistic }}-\delta v_{\text {Fermi }}= & \left(\delta v_{\text {realistic }}-\delta \nu_{\text {Fermi }}^{\text {def }}\right) \\
& +\left(\delta v_{\text {Fermi }}^{\text {def }}-\delta v_{\text {Fermi }}^{\text {sph }}\right) .
\end{aligned}
$$

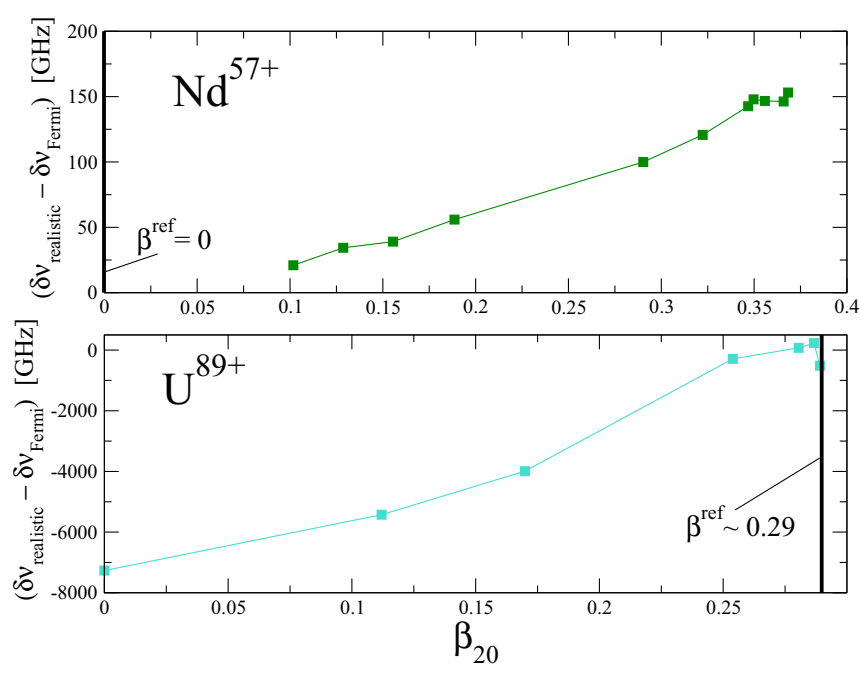

FIG. 5. The corrections $\delta v_{\text {realistic }}-\delta v_{\text {Fermi }}$ to the line field shift values as a function of the quadrupole deformation parameter $\beta_{20}$ of the target $A^{\prime}$ isotope for various (a) $\mathrm{Nd}^{57+}$ and (b) $\mathrm{U}^{89+}$ isotope pairs. In each case, the corresponding deformation of the reference isotope $A$ is indicated by a vertical line on the plots. All plotted values refer to the same resonance transition as in Fig. 4. 


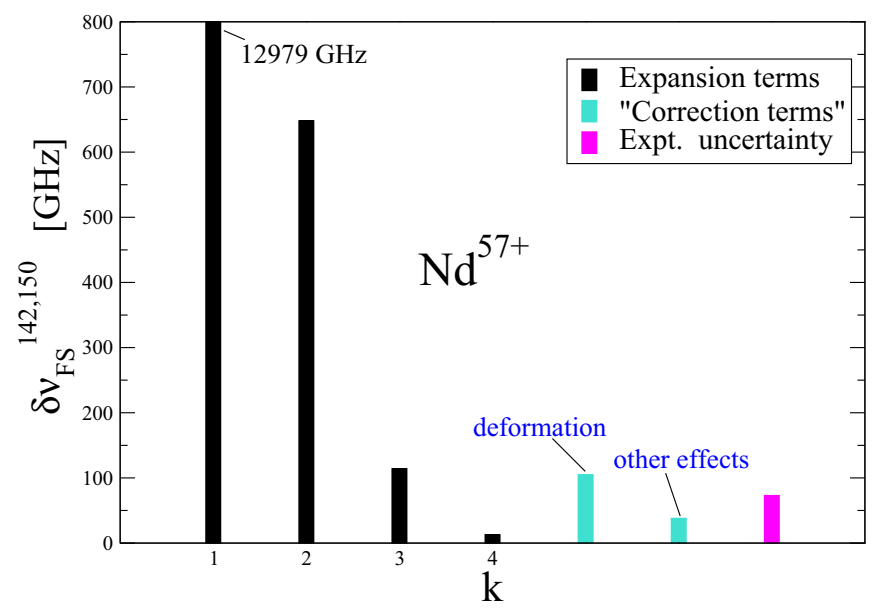

FIG. 6. Decomposition of expansion and correction terms of the $1 s^{2} 2 s^{2} S_{1 / 2} \longrightarrow 1 s^{2} 2 p^{2} P_{1 / 2}^{o}$ transition in Li-like ${ }^{142,150} \mathrm{Nd}$.

The $\delta v_{\text {Fermi }}^{\text {def }}-\delta v_{\text {Fermi }}^{\text {sph }}$ part isolates the effect of deformation, while the remaining $\delta v_{\text {realistic }}-\delta v_{\text {Fermi }}^{\text {def }}$ part gives the corrections due to "other effects," such as density wiggles and differences in diffuseness. In order to separately estimate the effect of deformation in Li-like Nd, the deformed Fermi model was used with $\beta_{20}$ values obtained from the microscopic nuclear calculations.

Isotope shift (IS) measurements have been performed for the first two resonance transitions of the ${ }^{142,150} \mathrm{Nd}^{57+}$ pair [37] and the statistical uncertainty of the observed isotope shift for the $1 s^{2} 2 s^{2} S_{1 / 2} \longrightarrow 1 s^{2} 2 p^{2} P_{1 / 2}^{o}$ transition is compared to the magnitude of the "correction terms" in Fig. 6. As seen in the figure, the effect of deformation is large enough to be detected by the experiments and the correction due to "other effects" is not negligible.

\section{B. Neutral atoms}

In this section, field shifts in neutral barium are investigated for the three well-known $6 s^{2}{ }^{1} S_{0} \longrightarrow 6 s 6 p^{1,3} P_{1}^{o}$ and $6 s^{2} S_{0} \longrightarrow 6 p^{2} P_{1}$ transitions. Figure 7 illustrates the dependence of the magnitude of the corrections on the deformation parameter $\beta_{20}$. The same trend is seen for the three transitions. As already deduced for $\mathrm{Nd}^{57+}$ and $\mathrm{U}^{89+}$ (see Fig. 5) the magnitude of $\delta v_{\text {realistic }}-\delta v_{\text {Fermi }}$ increases as the difference between the deformation of reference and target isotope becomes large. However, in neutral barium the magnitude of the correction term $\delta v_{\text {realistic }}-\delta v_{\text {Fermi }}$ is a factor $\sim 10^{3}$ smaller.

In contrast to the IS measurements in Li-like systems, a greater number of measurements has been performed in neutral atomic systems. Furthermore, in such measurements the accuracy provided is generally much higher. Following the process described in the previous section, the correction term is decomposed for the $6 s^{2} S_{0} \longrightarrow 6 s 6 p{ }^{1} P_{1}^{o}$ transition of the ${ }^{138,136} \mathrm{Ba}$ isotope pair. The isotope shift measurements of the corresponding spectral lines [34] carries a statistical error, which is in Fig. 8 compared to the magnitude of the correction terms.

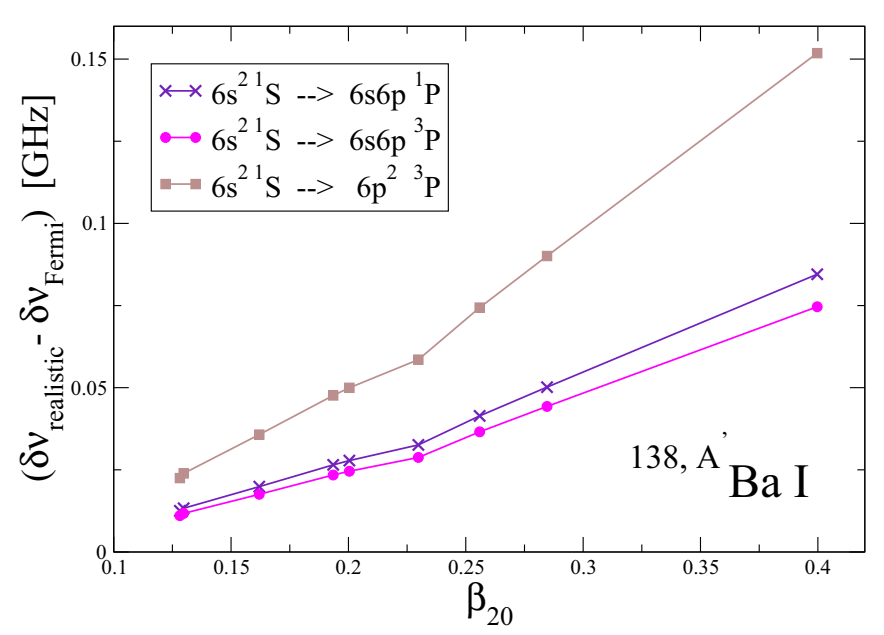

FIG. 7. The corrections $\delta v_{\text {realistic }}-\delta v_{\text {Fermi }}$ to the line field shift calculations as a function of the quadrupole deformation parameter $\beta_{20}$ of the target $A^{\prime}$ isotope for the neutral barium isotope pairs.

As seen in Fig. 8, the experimental uncertainty is remarkably small in comparison to the magnitude of the corrections. However, in reality the experimental uncertainty of the field shift is much larger since the theoretical mass shift contribution is in this case associated with large uncertainties, which are not reflected in this figure. The dominating corrections are the "other effects" that arise from the differences between the deformed Fermi distribution and the more realistic charge distributions obtained from the microscopic nuclear calculations.

The major improvement to the line field shift measurements illustrated in Fig. 3 is clearly due to the choice of using realistic rms radii. However, making in addition use of realistic higherorder nuclear moments leads to a non-negligible improvement in the description of the experimental data. According to the current experimental precision in the measurement of the isotope shifts in ${ }^{136,138} \mathrm{Ba}$ and ${ }^{150,142} \mathrm{Nd}^{57+}$, effects like deformation captured by the higher nuclear moments could be detected (see Figs. 6 and 8). As a result, information about such

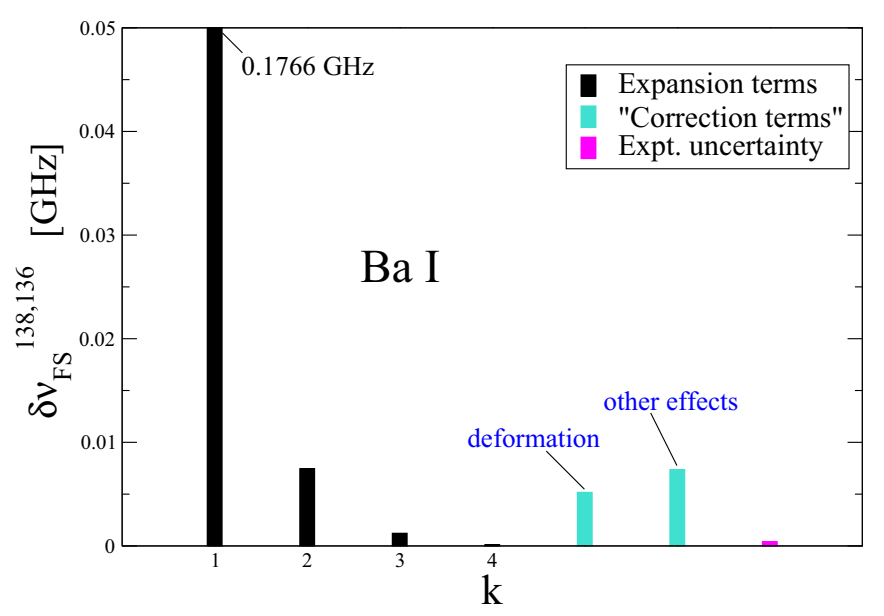

FIG. 8. Decomposition of expansion and correction terms of the $6 s^{2}{ }^{1} S_{0} \longrightarrow 6 s 6 p^{1} P_{1}^{o}$ transition in ${ }^{138,136} \mathrm{Ba} \mathrm{I}$. 
a nuclear property could possibly be deduced from isotope shift observations.

\section{V. $\delta\left\langle r^{4}\right\rangle^{A, A^{\prime}}$ EXTRACTION}

The nuclear charge radius is one of the most obvious and fundamental parameters, related to the size of the nucleus. Considering isotope shift measurements, the charge radii of an isotope sequence are typically determined in terms of the differences in the second radial moment $\delta\left\langle r^{2}\right\rangle$, between target isotope $A^{\prime}$ and reference isotope $A$. In contrast to light nuclei, in heavy nuclear systems the contribution of the higher-order radial moments to the line field shift can be significant and above the observable limit (see Figs. 6 and 8). Moreover, in highly charged heavy systems the contribution of the mass shift effect becomes smaller. This suggests the possibility to extract information about higher nuclear moments.

The reformulation of the field shift, combined with experimental isotope shift measurements, in principle enables the extraction of differences in higher-order radial moments $\delta\left\langle r^{2 N}\right\rangle, N=2,3,4$. Consequently, information about the nuclear shapes, deformations, density wiggles, and other nuclear properties can be provided. The extraction of all four radial moments requires four transitions $k$ to be available. A system of four equations is then solved for

$$
\begin{aligned}
\delta v_{k, \mathrm{RFS}}= & F_{k, 1} \delta\left\langle r^{2}\right\rangle+F_{k, 2} \delta\left\langle r^{4}\right\rangle \\
& +F_{k, 3} \delta\left\langle r^{6}\right\rangle+F_{k, 4} \delta\left\langle r^{8}\right\rangle,
\end{aligned}
$$

where $k=1,2,3,4$. However, it is rare that observed isotope shifts are available for four transitions and, in addition, such systems of equations cannot be formed so that they give trustworthy solutions for higher than second-order moments.

\section{A. RFS expansion using orthogonal moments}

As seen in Figs. 6 and 8, all four expansion terms do not equally contribute to the final field shift value. Considering in Fig. 9 the line field shift for the ${ }^{208,200} \mathrm{~Pb}$ pair, the

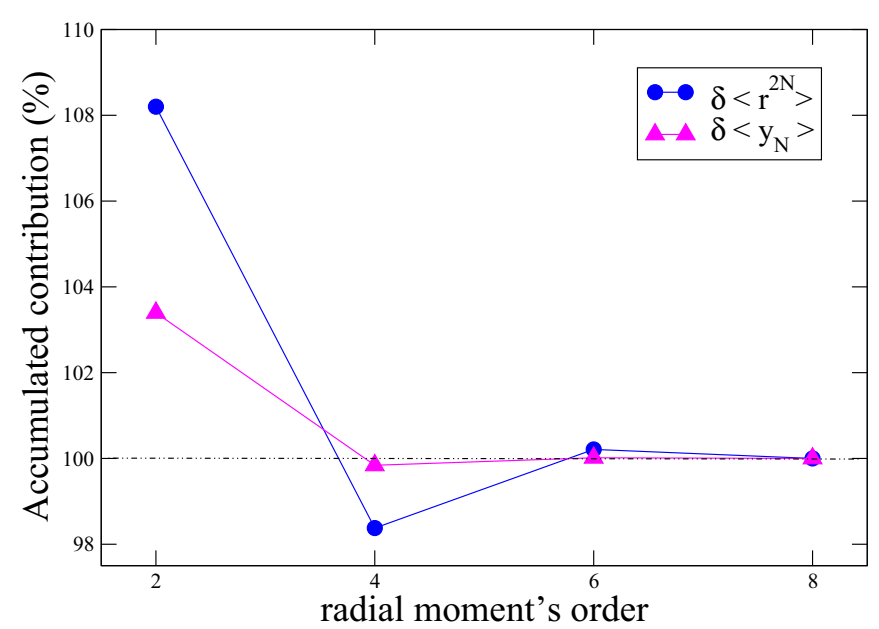

FIG. 9. $\sum_{N} F_{N} \delta\left\langle r^{2 N}\right\rangle / \delta v$ in percent (circles) compared to the corresponding expression for the rearranged summation (triangles). The plot refers to the ${ }^{208,200} \mathrm{~Pb}$ pair and the $1 s^{2} 2 s^{2} S_{1 / 2} \longrightarrow 1 s^{2} 2 p^{2} P_{1 / 2}^{o}$ transition. fourth-order radial moment adds $\sim 10 \%$ contribution, the sixth moment $\sim 2 \%$, and the last term, which contains the eighth-order moment, contributes with much less. Thus, it is fair to say that the major correction to the approximation that assumes constant electron density $\rho_{i}^{e}(\boldsymbol{r}) \approx \rho_{i}^{e}(0)$ comes from the second expansion term, i.e., $F_{k, 2} \delta\left\langle r^{4}\right\rangle$, which takes into account the differences between the $\left\langle r^{4}\right\rangle$ moments. However, the contribution from higher-order terms is not negligible.

In Eq. (20), the information about the nuclear charge distribution is encoded in a set of nuclear radial moments. These moments are not independent and a faster converging series may be found by instead expanding in a set of orthogonal polynomials (see Appendix). The convergence of this rearranged summation compared with the original summation is shown in Fig. 9. By taking into account only the first term, the line field shift is already much closer to the final value. The second term adds $\sim 3.5 \%$ contribution, the third $\sim 0.18 \%$, while the last one adds $\sim 0.016 \%$. Thus, accurate enough field shift predictions can now be provided using only the first two expansion terms containing the differences $\delta\left\langle y_{1}\right\rangle$ and $\delta\left\langle y_{2}\right\rangle$, which are in turn given as a function of the $\delta\left\langle r^{2}\right\rangle$ and $\delta\left\langle r^{4}\right\rangle$ moments (see Appendix). Having only two unknowns means that $\delta\left\langle r^{2}\right\rangle$ and $\delta\left\langle r^{4}\right\rangle$ can potentially be extracted from knowledge of two observed line field shifts in an isotope pair.

\section{B. Testing the method}

After expanding in the orthonormal basis, for a pair of isotopes $A, A^{\prime}$, the reformulated line field shift can to a very good approximation be expressed as

$$
\delta v_{k, \mathrm{RFS}} \approx c_{k, 1} \delta\left\langle y_{1}\right\rangle+c_{k, 2} \delta\left\langle y_{2}\right\rangle
$$

where the $c_{k, 1}$ and $c_{k, 2}$ coefficients are expressed in terms of the $F_{k, N}$ factors. In order to test the method, theoretical line field shifts $\delta \nu_{\text {RFS }}$ were obtained using realistic nuclear radial moments. These line field shifts refer to the $1 s^{2} 2 s^{2} S_{1 / 2} \longrightarrow$ $1 s^{2} 2 p^{2} P_{1 / 2}^{o}$ and $1 s^{2} 2 s^{2} S_{1 / 2} \longrightarrow 1 s^{2} 2 p^{2} P_{3 / 2}^{o}$ transitions of the uranium, lead, and neodymium isotope pairs studied in Sec. IV. Using these calculated field shifts as "pseudoexperimental" input data, the equations can be inverted and should yield, if the method is flawless, extracted radial moments which are identical to the realistic nuclear moments used in the computation of the field shifts.

In all cases, the extracted $\delta\left\langle r^{2}\right\rangle$ moments are almost identical to the exact $\delta\left\langle r^{2}\right\rangle_{\text {realistic moments. The difference is }}$ less than $0.0002 \mathrm{fm}^{2}$ for all lead and uranium isotopes, as well as the neodymium isotopes that are close to spherical. For the highly deformed neodymium isotopes, the difference is slightly larger, of the order of $\sim 0.001 \mathrm{fm}^{2}$, which still represents a small discrepancy.

In Fig. 10, the extracted $\delta\left\langle r^{4}\right\rangle$ values have been plotted and compared to the $\delta\left\langle r^{4}\right\rangle_{\text {realistic }}$ representing exact values. The extracted $\delta\left\langle r^{4}\right\rangle_{\text {original }}$ values using the first two terms of the original summation $\delta v_{k, \mathrm{RFS}}^{A, A^{\prime}} \approx \sum_{N=1}^{2} F_{k, N} \delta\left\langle r^{2 N}\right\rangle$ are in addition illustrated in the same figure. When the rearranged summation is used, the extracted $\delta\left\langle r^{4}\right\rangle$ moments are in good agreement with the exact $\delta\left\langle r^{4}\right\rangle_{\text {realistic moments, whereas the }}$ $\delta\left\langle r^{4}\right\rangle$ moments using the original, but truncated, summation display an observable discrepancy from the exact values. All in all, the expression using the rearranged summation for 


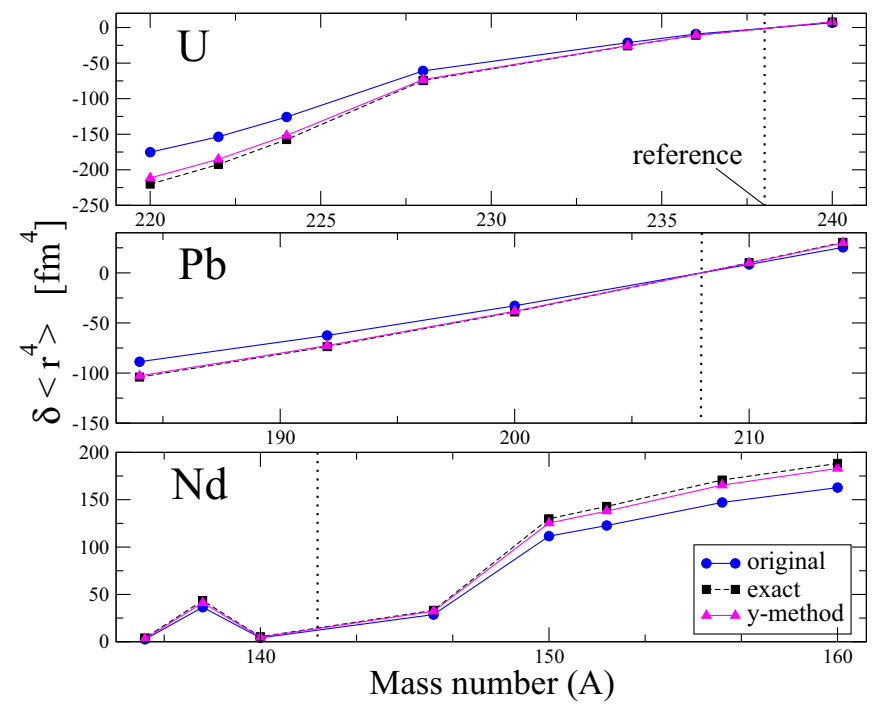

FIG. 10. The extracted $\delta\left\langle r^{4}\right\rangle$ values for the uranium, lead, and neodymium isotope pairs, previously studied. The dashed line with squares represents the exact $\delta\left\langle r^{4}\right\rangle_{\text {realistic }}$ values, obtained from the HFB and HFBTHO calculations for the spherical lead and the deformed uranium and neodymium isotopes, respectively. The line with triangle symbols represents the extracted $\delta\left\langle r^{4}\right\rangle$ when $\delta v_{k, \mathrm{FS}} \approx c_{k, 1} \delta\left\langle y_{1}\right\rangle+$ $c_{k, 2} \delta\left\langle y_{2}\right\rangle$ is assumed, while the line with circles corresponds to the $\delta\left\langle r^{4}\right\rangle_{\text {original }}$ extracted when the original summation in $\delta v_{k, \mathrm{RFS}}$ has been used.

the reformulated field shift enables the determination of the differences between $r^{2}$ and $r^{4}$ moments, much more accurately than using the original expression.

\section{Towards the extraction of $\delta\left\langle r^{2}\right\rangle$ and $\delta\left\langle r^{4}\right\rangle$ moments using experimental data}

In what follows, the major objective is to discuss how $\delta\left\langle r^{2}\right\rangle$ and $\delta\left\langle r^{4}\right\rangle$ moments can be extracted from experimental data using the method tested above. From observed isotope shifts, experimental field shift values can be obtained by estimating and removing the mass shift contribution and residual effects $\delta v_{k, \text { RES }}$ from for example QED and nuclear polarization (see, e.g., [6]):

$$
\delta v_{k, \mathrm{FS}}^{\mathrm{expt}}=\delta v_{k, \mathrm{IS}}^{\mathrm{expt}}-\delta v_{k, \mathrm{MS}}-\delta v_{k, \mathrm{RES}} .
$$

The effect from nuclear polarization is not estimated in this work, but have been studied extensively in the past by Plunien et al. [39-42]. For Li-like systems, the contribution is comparable to the effect from deformation and must be taken into account in real situations.

Without making use of RFS, the difference in $\left\langle r^{2}\right\rangle$ moments can now be extracted by performing variational calculations where the rms radius of the reference isotope is estimated and $\delta\left\langle r^{2}\right\rangle$ is varied until agreement with experimental field shifts is observed (see, for example, [37]):

$$
\delta v_{k, \mathrm{FS}}^{\text {expt }}=\delta v_{k, \mathrm{VA}}^{\text {exact }} .
$$

The difference in higher moments then follows from the model used to mimic the nuclear charge distribution, for example the Fermi distribution, and hence this method is highly model
TABLE II. The line frequency field shift values, resulting from the variational calculations using GRASP2K and the reformulation of the field shift, are respectively displayed for a few lead and uranium isotope pair combinations. In the last column, the discrepancy between $\delta v_{\mathrm{VA}}^{\text {exact }}$ and $\delta v_{\mathrm{RFS}}$ is computed. "Transition 1 " refers to the $1 s^{2} 2 s^{2} S_{1 / 2} \longrightarrow 1 s^{2} 2 p^{2} P_{1 / 2}^{o}$ transition.

\begin{tabular}{lccc}
\hline \hline Transition 1 & $\delta \nu_{\mathrm{VA}}^{\text {exact }}(\mathrm{GHz})$ & $\delta v_{\mathrm{RFS}}(\mathrm{GHz})$ & $d(\mathrm{GHz})$ \\
\hline 208,192 & 51303 & 50563 & 740 \\
208,200 & 28938 & 28546 & 392 \\
208,210 & -14186 & -14021 & -165 \\
238,234 & 54796 & 53976 & 820 \\
238,236 & 27412 & 27015 & 397 \\
\hline \hline
\end{tabular}

dependent. However, making use of the reformulation of field shifts using an orthogonal moments basis, we instead use experimental field shift values from two transitions and solve the following equation system in order to extract the $\delta\left\langle r^{2}\right\rangle$ and $\delta\left\langle r^{4}\right\rangle$ moments virtually model independent:

$$
\delta \nu_{k, \mathrm{FS}}^{\mathrm{expt}}=c_{k, 1} \delta\left\langle y_{1}\right\rangle+c_{k, 2} \delta\left\langle y_{2}\right\rangle+d_{k} .
$$

In the expression above, a term $d_{k}$ has been introduced which represents the discrepancy between the "exact" variational solution $\delta v_{k, \mathrm{VA}}^{\text {exact}}$, and the RFS solution $\delta v_{k, \mathrm{RFS}}$, assuming a spherical Fermi nuclear charge distribution for the reference and the target isotope. To examine the importance of the $d_{k}$ term for the extraction of the radial moments, we used GRASP2K and RIS4 to compute $\delta v_{k, \mathrm{VA}}^{\text {exact }}$ and $\delta v_{k, \mathrm{RFS}}$ for the resonance transitions in several Li-like lead and uranium isotope pairs. In the calculations, rms radii were taken from the compilation by Angelis and Marinova [1] and the results are presented in Tables II and III. As seen, an expected discrepancy between the $\delta v_{\mathrm{VA}}^{\text {exact }}$ and $\delta \nu_{\mathrm{RFS}}$ values, i.e., the $d_{k}$ term, is observed for both transitions. In our case, this discrepancy is mainly due to QED effects included in the VA calculation that become important in heavy nuclei and which are not included in the perturbative approach. In addition, these QED contributions (vacuum polarization and self-energy) depend on the nuclear size [43] and hence the $d_{k}$ terms should be reevaluated when the nuclear parameters of the isotopes are changed. Other assumptions that have been made throughout the formulation of the perturbative approach are expected to play a minor role. Indicatively, for "transition 1" in the uranium isotope pairs the magnitude of the discrepancy is of the order of $\sim 1.5 \%$ of the $\delta \nu_{\mathrm{VA}}^{\text {exact }}$ value, from which $\sim 0.1 \%$ is due to other than QED effects. It is also seen that the $d_{k}$ terms for the two transitions are slightly different, and it turns out that accurately estimating this difference, rather than the magnitude of the

TABLE III. Same as Table II. "Transition 2" refers to the $1 s^{2} 2 s^{2} S_{1 / 2} \longrightarrow 1 s^{2} 2 p^{2} P_{3 / 2}^{o}$ transition.

\begin{tabular}{lccc}
\hline \hline Transition 2 & $\delta v_{\mathrm{VA}}^{\text {exact }}(\mathrm{GHz})$ & $\delta v_{\mathrm{RFS}}(\mathrm{GHz})$ & $d(\mathrm{GHz})$ \\
\hline 208,192 & 55459 & 54642 & 817 \\
208,200 & 31282 & 30848 & 434 \\
208,210 & -15336 & -15152 & -184 \\
238,234 & 61189 & 60277 & 912 \\
238,236 & 30610 & 30169 & 441 \\
\hline \hline
\end{tabular}


terms, is absolutely crucial in order to extract accurate $\delta\left\langle r^{4}\right\rangle$ moments.

We are now ready to show that it is possible to extract $\delta\left\langle r^{2}\right\rangle$ and $\delta\left\langle r^{4}\right\rangle$ moments if accurate experimental field shifts are available. This is due to the fact that the electronic factors $c_{k}$ and the $d_{k}$ terms can be accurately estimated also when the rms radii is not known for the reference and/or target isotope. In these cases, we make instead a "qualified guess" for the rms radii. The parametrization, given in Eq. (14), for the rms radius of an isotope $A$ constitutes an example of such a "qualified guess" and will be used below.

\section{Root-mean-square radii data available for the reference isotopes}

When radial moment differences are deduced from isotope shift measurements, the nuclear parameters are usually known for the reference isotope but not for the target isotope. We will now demonstrate the procedure for how experimental $\delta\left\langle r^{2}\right\rangle$ and $\delta\left\langle r^{4}\right\rangle$ moments for the ${ }^{238,234} \mathrm{U}$ isotope can be extracted in such cases by considering the two resonance transitions in Li-like uranium. In what follows, ${ }^{238} \mathrm{U}$ is the reference isotope, $r_{A}^{\text {tab }}$ denote a tabulated rms radius for isotope $A$ taken from [1], $r_{A}^{\text {para }}$ denote a parametrized rms radius for isotope $A$ using Eq. (14), and spherical Fermi distributions with $t=2.30 \mathrm{fm}$ are used everywhere. Further on it is assumed that accurate $\delta v_{k, \mathrm{FS}}^{\text {expt }}$ values are available:

(1) Two separate variational calculations are performed using $r_{238}^{\text {tab }}=5.8571 \mathrm{fm}$ and $r_{234}^{\text {para }}=5.7216 \mathrm{fm}$, respectively.

(2) $\delta v_{k, \mathrm{VA}}^{\text {exact }}$ is constructed using the level energies from the $r_{238}^{\text {tab }}$ and $r_{234}^{\text {para }}$ calculations in step 1 .

(3) $\delta v_{k, R F S}$ is computed by using the electronic factors from the $r_{238}^{\mathrm{tab}}$ calculation and the difference in radial moments as predicted by two spherical Fermi distributions with $r_{238}^{\text {tab }}$ and $r_{234}^{\text {para }}$, respectively.

(4) $d_{k}=\delta v_{k, \mathrm{VA}}^{\text {exact }}-\delta v_{k, \mathrm{RFS}}$ is computed.

(5) $c_{k}$ factors are computed using the electronic factors in step 3 (see Appendix).

(6) $\delta\left\langle y_{1}\right\rangle$ and $\delta\left\langle y_{2}\right\rangle$ are extracted by solving Eq. (24).

(7) $\delta\left\langle r^{2}\right\rangle$ and $\delta\left\langle r^{4}\right\rangle$ are computed (see Appendix).

To quantitatively validate the method we replace $\delta v_{k, \mathrm{FS}}^{\text {expt }}$ with "pseudoexperimental" field shifts constructed from two separate variational calculations using $r_{238}^{\mathrm{tab}}$ and $r_{234}^{\mathrm{tab}}=5.8291$ $\mathrm{fm}$, respectively. In addition, we repeat the procedure for the ${ }^{238,236} \mathrm{U}$ isotope pair using $r_{236}^{\text {para }}=5.7363 \mathrm{fm}$ and $r_{236}^{\text {tab }}=$ $5.8431 \mathrm{fm}$. In Table IV, the extracted $\delta\left\langle r^{2}\right\rangle$ and $\delta\left\langle r^{4}\right\rangle$ moments

TABLE IV. Errors, in $\mathrm{fm}^{2}$ and $\mathrm{fm}^{4}$, when extracting the $\delta\left\langle r^{2}\right\rangle$ and $\delta\left\langle r^{4}\right\rangle$ moments, for the ${ }^{234,238} \mathrm{U}$ and ${ }^{236,238} \mathrm{U}$ pairs. It is assumed that the rms radii are unknown for the target isotopes. See text for details.

\begin{tabular}{lrr}
\hline \hline & 238,234 & \multicolumn{1}{c}{238,236} \\
\hline$\delta\left\langle r^{2}\right\rangle$ & -0.3282 & -0.1642 \\
$\delta\left\langle r^{2}\right\rangle_{\text {expt }}$ & -0.3272 & -0.1638 \\
Error & 0.0010 & 0.0004 \\
$\delta\left\langle r^{4}\right\rangle$ & -28.9026 & -14.3453 \\
$\delta\left\langle r^{4}\right\rangle_{\text {expt }}$ & -27.4419 & -13.7693 \\
Error & 1.4607 & 0.5760 \\
\hline \hline
\end{tabular}

are compared to the experimental $\delta\left\langle r^{2}\right\rangle_{\text {expt }}$ and $\delta\left\langle r^{4}\right\rangle_{\text {expt }}$ moments. As seen, the extracted $\delta\left\langle r^{2}\right\rangle$ moments are almost identical to the "experimental" values. In addition, the $\delta\left\langle r^{4}\right\rangle$ moments are extracted with an accuracy of $5.3 \%$ and $4.2 \%$ for the ${ }^{234,238} U$ and ${ }^{236,238} U$ pairs, respectively. The errors, which are of systematical nature and remarkably small, arise from estimating the $d$ term using rms radii for the target isotopes which differ by approximately $0.11 \mathrm{fm}$ from the tabulated values used to construct the "pseudoexperimental" field shifts. However, after the extraction one obtains a better estimate for the rms radii of the target isotopes that allows the method to be iteratively improved.

\section{Root-mean-square radii unknown for both target and reference isotopes}

Assuming that the rms radius value of the reference isotope is also unknown, we again try to extract the $\delta\left\langle r^{2}\right\rangle$ and $\delta\left\langle r^{4}\right\rangle$ moments. A "qualified guess" for the rms radius of ${ }^{238} \mathrm{U}$ is then needed and we replace $r_{238}^{\mathrm{tab}}$ with $r_{238}^{\mathrm{para}}=5.7508 \mathrm{fm}$ in the procedure described above.

The results from the extraction of the $\delta\left\langle r^{2}\right\rangle$ and $\delta\left\langle r^{4}\right\rangle$ moments are presented in Table V. As seen, the $\delta\left\langle r^{2}\right\rangle$ moment is extracted almost as accurate as before (see Table IV). Further on, the results from extracting the $\delta\left\langle r^{4}\right\rangle$ moments display a discrepancy of $\sim 10.3 \%$ and $\sim 6.5 \%$ from the exact values, for the ${ }^{234,238} \mathrm{U}$ and ${ }^{236,238} \mathrm{U}$ pairs, respectively.

The nuclear parameters relevant to the reference isotope have been modified here. Thus, the $F_{k}$ factors have also been reevaluated since they are always deduced for the reference isotope. As a result, aside from the new radial moments differences, the $\delta v_{k, \text { RFS }}$ field shifts are computed based on updated sets of $F_{k, N}$ factors. This explains the larger discrepancy that is observed when extracting the $\delta\left\langle r^{2}\right\rangle$ and $\delta\left\langle r^{4}\right\rangle$ moments in the latter case (see Table V). However, the results are remarkably good given that the "qualified guess" for the reference isotope is approximately $0.11 \mathrm{fm}$ smaller than the tabulated value used to construct the pseudoexperimental field shifts.

\section{Statistical errors when extracting the $\delta\left\langle r^{2}\right\rangle$ and $\delta\left\langle r^{4}\right\rangle$ moments}

Above, the $\delta\left\langle r^{2}\right\rangle$ and $\delta\left\langle r^{4}\right\rangle$ moments were extracted by solving the matrix equation

$$
\left[\begin{array}{l}
\delta v_{1, \mathrm{RFS}} \\
\delta v_{2, \mathrm{RFS}}
\end{array}\right]=C\left[\begin{array}{l}
\delta\left\langle y_{1}\right\rangle \\
\delta\left\langle y_{2}\right\rangle
\end{array}\right] .
$$

TABLE V. Same as Table IV. Here, it is assumed that rms radii are unknown for both the reference and the target isotopes. See text for details.

\begin{tabular}{lrr}
\hline \hline & 238,234 & \\
\hline$\delta\left\langle r^{2}\right\rangle$ & -0.3287 & -0.1640 \\
$\delta\left\langle r^{2}\right\rangle_{\text {expt }}$ & -0.3272 & -0.1638 \\
Error & 0.0015 & 0.0002 \\
$\delta\left\langle r^{4}\right\rangle$ & -30.2665 & -14.6612 \\
$\delta\left\langle r^{4}\right\rangle_{\text {expt }}$ & -27.4419 & -13.7693 \\
Error & 2.8246 & 0.8919 \\
\hline \hline
\end{tabular}


In order to solve for $y_{1}$ and $y_{2}$, the matrix $C$ must be invertible. If the matrix determinant is zero, then the matrix is singular and cannot be inverted. It is not rare that the determinant of such matrix can be close to zero, but still nonzero. In this case, the matrix is close to singular and as a result the values of $\delta\left\langle y_{1}\right\rangle$ and $\delta\left\langle y_{2}\right\rangle$ will be hugely affected, even by a small change in the field shifts $\delta v_{1, \text { RFS }}$ and $\delta v_{2, \text { RFS }}$. Namely, the extracted $\delta\left\langle y_{1}\right\rangle$ and $\delta\left\langle y_{2}\right\rangle$ values, and as a consequence the $\delta\left\langle r^{2}\right\rangle$ and $\delta\left\langle r^{4}\right\rangle$ moments, will to a great degree be affected by the uncertainties in the observed isotope shifts, making the extraction of the radial nuclear moments with high accuracy a difficult task. A $C$-matrix determinant equal to zero is obtained if the two equations are linearly dependent. In such case, it is not possible to extract two unknowns. Therefore, the transitions considered should be as independent as possible in terms of electronic factors.

The observed isotope shifts $\delta v_{k, I S}^{\text {expt }}$, and subsequently the observed field shifts $\delta v_{k, \mathrm{FS}}^{\text {expt }}$, are associated with uncertainties of a certain magnitude. These uncertainties lead to statistical errors in the extracted nuclear moments. In the next subsections, the propagation of these errors is discussed and how they can be minimized by selecting atomic transitions.

\section{Statistical errors in relation to the atomic number}

In Sec. V B, our method was tested by using $\delta v_{\mathrm{RFS}}$ line field shifts as pseudoexperimental data. In order to extend this approach to consider uncertainties we assume uncorrelated errors with an uncertainty $\pm \epsilon$, where $\epsilon=\delta v_{k, \mathrm{RFS}} \times 10^{-m}$, in the $\delta v_{k, \mathrm{RFS}}$ values that are used for solving the matrix equation [see Eq. (25)]. By varying $m$, the magnitude of the field shift uncertainty changes. We can then investigate the effect these uncertainties have on the extracted $\delta\left\langle r^{2}\right\rangle$ and $\delta\left\langle r^{4}\right\rangle$ values.

The extraction of the $\delta\left\langle r^{2}\right\rangle$ and $\delta\left\langle r^{4}\right\rangle$ moments was in Sec. V B performed for several uranium, lead, and neodymium isotope pairs (see Fig. 10). By making a reasonable choice of $m=3$ for the error $\epsilon$ in the $\delta v_{k, \text { RFS }}$ values relevant to these isotope pairs, it is possible to estimate the magnitude of the statistical errors in the extracted $\delta\left\langle r^{2}\right\rangle$ and $\delta\left\langle r^{4}\right\rangle$ moments. The relative errors of the extracted values for one isotope pair of each of the above elements are indicatively presented in Table VI. The error in $\delta\left\langle r^{2}\right\rangle^{142,150}$ is approximately $72 \%$ of the magnitude of the resulting value. Besides, the $\delta\left\langle r^{4}\right\rangle^{142,150}$ is extracted with significantly greater error. However, the relative error in both $\delta\left\langle r^{2}\right\rangle$ and $\delta\left\langle r^{4}\right\rangle$ demonstrates a considerable decrease as the atomic number of the isotopes becomes larger.

TABLE VI. The relative error in the extraction of the $\delta\left\langle r^{2}\right\rangle$ and $\delta\left\langle r^{4}\right\rangle$ moments for the ${ }^{142,150} \mathrm{Nd}^{57+},{ }^{208,192} \mathrm{~Pb}^{79+}$, and ${ }^{238,236} \mathrm{U}^{89+}$ pairs. The relative errors are presented as a function of the atomic number of these three elements. The inaccuracy assumed in the $\delta v_{k, \mathrm{RFS}}$ field shift data is $\pm \epsilon=\delta v_{k, \mathrm{RFS}} \times 10^{-3}$.

\begin{tabular}{lccc}
\hline \hline & ${ }^{142,150} \mathrm{Nd}$ & ${ }^{208,192} \mathrm{~Pb}$ & ${ }^{238,236} \mathrm{U}$ \\
$Z$ & 60 & 82 & 92 \\
\hline$\frac{\Delta\left(\delta\left\langle r^{2}\right\rangle\right)}{\left|\delta\left\langle r^{2}\right\rangle\right|}$ & 0.72 & 0.39 & 0.28 \\
$\frac{\Delta\left(\delta\left\langle r^{4}\right\rangle\right)}{\left|\delta\left\langle r^{4}\right\rangle\right|}$ & 13.84 & 5.54 & 3.65 \\
\hline \hline
\end{tabular}

So far, the extraction of the $\delta\left\langle r^{2}\right\rangle$ and $\delta\left\langle r^{4}\right\rangle$ moments was performed by making use of $\delta v_{k, \text { RFs }}$ field shifts and $F_{k, N}$ line field shift factors that are attributed to the first two resonance transitions, i.e., $1 s^{2} 2 s^{2} S_{1 / 2} \longrightarrow 1 s^{2} 2 p^{2} P_{1 / 2}^{o}$ and $1 s^{2} 2 s^{2} S_{1 / 2} \longrightarrow 1 s^{2} 2 p^{2} P_{3 / 2}^{o}$. For these two transitions in lithium-like systems, the $F_{k, N}$ factors, as well as the line mass shift parameters $\Delta K_{k, \mathrm{MS}}$, can be determined with high accuracy. Therefore, when we in practice attempt to extract the $\delta\left\langle r^{2}\right\rangle$ and $\delta\left\langle r^{4}\right\rangle$ moments using actual experimental data, the uncertainties in the $\delta v_{k, \mathrm{FS}}^{\text {expt }}$ values will normally be dominated by the uncertainties in the $\delta v_{k, \text { IS }}^{\text {expt }}$ measurements.

For the ${ }^{142,150} \mathrm{Nd}^{57+}$ pair and the previously mentioned transitions such measurements are available [37]. Taking into account the uncertainties in the measured isotope shifts $\delta v_{k, \text { IS }}^{\text {expt }}$, the corresponding uncertainties in $\delta v_{k, \mathrm{FS}}^{\text {expt }}$ appear in the fourth and third digits for each of the above transitions, respectively. In this case, the choice of an error $\pm \epsilon=\delta v_{k, \mathrm{RFS}} \times 10^{-3}$ in the calculated field shift values seems to be quite realistic. However, according to Table VI the errors in the $\delta\left\langle r^{2}\right\rangle$ and $\delta\left\langle r^{4}\right\rangle$ values resulting from experimental uncertainties of this magnitude for the neodymium pair are evidently extremely large.

We can therefore draw the conclusion that the extraction of the $\delta\left\langle r^{2}\right\rangle^{142,150}$ and $\delta\left\langle r^{4}\right\rangle^{142,150}$ moments with satisfactory accuracy is not likely to be a possibility at the moment. Varying $m$ we deduce that in order for the $\delta\left\langle r^{2}\right\rangle^{142,150}$ and $\delta\left\langle r^{4}\right\rangle^{142,150}$ to be determined with uncertainties of the order of $\lesssim 1 \%$ and $\lesssim 14 \%$, respectively, we should assume $m \geqslant 5$. In addition, considering Table VI, a more precise extraction of the $\delta\left\langle r^{2}\right\rangle$ and $\delta\left\langle r^{4}\right\rangle$ moments should be possible for the lead and in particular for the uranium isotope pairs.

\section{Independent transitions}

Considering the two resonance transitions that were used above for extracting $\delta\left\langle r^{2}\right\rangle$ and $\delta\left\langle r^{4}\right\rangle$ moments, we note that the same final state takes part in both. Therefore, these two transitions are not entirely independent and the corresponding $F_{k, N}$ factors do not constitute the best possible set so that we avoid matrix $C$ being close to singular. As a consequence, the uncertainties in the $\delta\left\langle r^{2}\right\rangle$ and $\delta\left\langle r^{4}\right\rangle$ values are relatively large. In order to be able to accurately extract both $\delta\left\langle r^{2}\right\rangle$ and $\delta\left\langle r^{4}\right\rangle$ moments, the precision of the experimental methods must therefore be improved substantially. Alternatively, a larger number of transitions must be available. Using the GRASP2K package, we can easily compute line field shift parameters for more transitions and hence an extended set of $\delta v_{k, \text { RFS }}$ values can be generated. The matrix equation will then be formed using $k>2$ equations, which need to be solved for the same unknowns $y_{1}$ and $y_{2}$. Having more equations than number of unknowns leads to a reduction of the statistical errors.

Choosing, for instance, to extract the $\delta\left\langle r^{2}\right\rangle$ and $\delta\left\langle r^{4}\right\rangle$ moments for the ${ }^{238,236} \mathrm{U}$ pair, we solve a matrix equation that consists of 16 equations corresponding to 16 different transitions. These transitions involve the following even $1 s^{2} 2 s^{2} S_{1 / 2}, \quad 1 s^{2} 3 s^{2} S_{1 / 2}, \quad 1 s^{2} 3 d{ }^{2} D_{3 / 2,5 / 2}$ and odd $1 s^{2} 2 p^{2} P_{1 / 2,3 / 2}^{o}, 1 s^{2} 3 p^{2} P_{1 / 2,3 / 2}^{o}$ states in Li-like uranium. By making the same choice of $m=3$ for the error $\epsilon=\delta v_{k, \text { RFS }} \times$ $10^{-m}$ in the $\delta v_{k, \text { RFS }}$ values, we extract the $\delta\left\langle r^{2}\right\rangle$ and $\delta\left\langle r^{4}\right\rangle$ moments. The extracted $\delta\left\langle r^{2}\right\rangle$ moment has exactly the same value 
TABLE VII. The relative error in the extraction of the $\delta\left\langle r^{2}\right\rangle$ and $\delta\left\langle r^{4}\right\rangle$ moments for the ${ }^{238,236} \mathrm{U}^{89+}$ pair, initially calculated when the first two resonance transitions were studied, when all 16 theoretically available transitions are used and when we finally choose one set of as independent as possible transitions. The uncertainties assumed in the $\delta v_{k, \mathrm{RFS}}$ field shift data are, as in Table VI, $\pm \epsilon=\delta v_{k, \mathrm{RFS}} \times 10^{-3}$.

\begin{tabular}{llll}
\hline \hline $238,236 \mathrm{U}$ & 2 res. & All 16 & 2 ind. \\
\hline$\frac{\Delta\left(\delta\left\langle r^{2}\right\rangle\right)}{\left|\delta\left\langle r^{2}\right\rangle\right|}$ & 0.28 & 0.03 & 0.02 \\
$\frac{\Delta\left(\delta\left\langle r^{4}\right\rangle\right)}{\left|\delta\left\langle r^{4}\right\rangle\right|}$ & 3.65 & 0.38 & 0.30 \\
\hline \hline
\end{tabular}

as before, whereas the $\delta\left\langle r^{4}\right\rangle$ value is also about the same, suffering from approximately the same systematical errors. However, the statistical errors in the extraction of both $\delta\left\langle r^{2}\right\rangle$ and $\delta\left\langle r^{4}\right\rangle$ have now been decreased significantly (see Table VII).

In practice, such large number of measured transitions is not likely to be available. Trying all different combinations, we realize that the error in the extraction of the $\delta\left\langle r^{2}\right\rangle$ and $\delta\left\langle r^{4}\right\rangle$ moments, by using a set of only two transitions, varies with the choice of the transitions. For the ${ }^{238,236} \mathrm{U}$ pair and $\epsilon=$ $\delta v_{k, \text { RFS }} \times 10^{-3}$ we get $0.0014 \leqslant \frac{\Delta\left(\delta\left\langle r^{2}\right\rangle\right)}{\left|\delta\left\langle r^{2}\right\rangle\right|} \leqslant 80$ and $0.0012 \leqslant$ $\frac{\Delta\left(\delta\left\langle r^{4}\right\rangle\right)}{\left|\delta\left\langle r^{4}\right\rangle\right|} \leqslant 1100$, for the relative errors in the extraction of the $\delta\left\langle r^{2}\right\rangle$ and $\delta\left\langle r^{4}\right\rangle$ moments, respectively.

We therefore deduce that in order to limit the magnitude of the statistical errors, it is more important to make a choice of as independent as possible transitions that form the set of equations solved, rather than increasing the number of transitions. Based on this conclusion, instead of extracting the $\delta\left\langle r^{2}\right\rangle$ and $\delta\left\langle r^{4}\right\rangle$ moments using the first two resonance transitions, a set of two more independent transitions is chosen. Thus, we attempt to extract the $\delta\left\langle r^{2}\right\rangle$ and $\delta\left\langle r^{4}\right\rangle$ moments for the ${ }^{238,236} \mathrm{U}$ isotope pair, using the resonance transition $1 s^{2} 2 s^{2} S_{1 / 2} \longrightarrow 1 s^{2} 2 p^{2} P_{1 / 2}^{o}$ combined with the $1 s^{2} 3 p^{2} P_{1 / 2}^{o} \longrightarrow 1 s^{2} 3 d^{2} D_{3 / 2}$ transition. The resulting relative errors for this combination of transitions are also displayed in Table VII. As seen, the relative errors in the extraction of both $\delta\left\langle r^{2}\right\rangle$ and $\delta\left\langle r^{4}\right\rangle$ moments are decreased when a more optimal combination of 2 out of the total 16 available transitions is chosen.

TABLE VIII. Same as Table IV. Here, the line field shift factors $F_{k, N}$ correspond to the $1 s^{2} 2 s^{2} S_{1 / 2} \longrightarrow 1 s^{2} 2 p{ }^{2} P_{1 / 2}^{o}$ and $1 s^{2} 3 p^{2} P_{1 / 2} \longrightarrow 1 s^{2} 3 d^{2} D_{3 / 2}$ transitions. Statistical errors are given in the rightmost column assuming uncertainties in the "pseudoexperimental" field shifts according to $\epsilon=\delta v_{k, \mathrm{RFS}} \times 10^{-3}$.

\begin{tabular}{lrr}
\hline \hline & 238,236 & \\
\hline$\delta\left\langle r^{2}\right\rangle$ & -0.1646 & \pm 0.0036 \\
$\delta\left\langle r^{2}\right\rangle_{\text {exact }}$ & -0.1638 & \\
Error & 0.0008 & \\
$\delta\left\langle r^{4}\right\rangle$ & -14.7283 & \pm 3.5279 \\
$\delta\left\langle r^{4}\right\rangle_{\text {exact }}$ & -13.7693 & \\
Error & 0.9590 & \\
\hline \hline
\end{tabular}

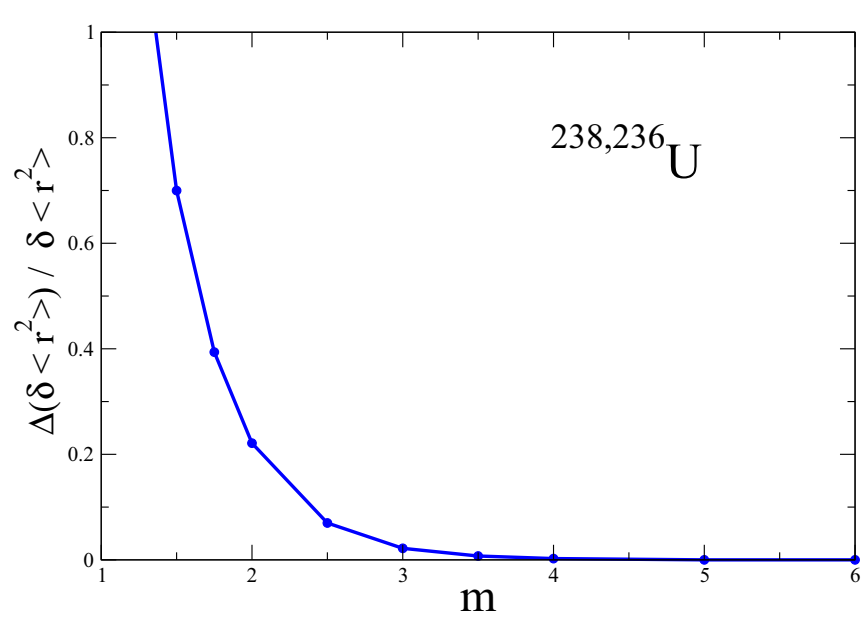

FIG. 11. The relative error in the extraction of the $\delta\left\langle r^{2}\right\rangle$ moment as a function of $m$ number in the assumed uncertainty $\epsilon=\delta v_{k, \mathrm{RFS}} \times$ $10^{-m}$ of the field shift. For the extraction, the pair of $1 s^{2} 2 s^{2} S_{1 / 2} \longrightarrow$ $1 s^{2} 2 p^{2} P_{1 / 2}^{o}$ and $1 s^{2} 3 p^{2} P_{1 / 2} \longrightarrow 1 s^{2} 3 d^{2} D_{3 / 2}$ transitions in Li-like ${ }^{238,236} \mathrm{U}$ has been used.

\section{Errors in the extraction of $\delta\left\langle r^{4}\right\rangle^{238,236}$}

Having ascertained that the "right" combination of transitions provides us with reasonably small statistical errors, we can extract the $\delta\left\langle r^{2}\right\rangle$ and $\delta\left\langle r^{4}\right\rangle$ moments for the ${ }^{238,236} \mathrm{U}$ isotope pair using pseudoexperimental field shifts, as described in Sec. V C 1, for this "optimal" pair of transitions. The statistical uncertainties are estimated as $\epsilon=\delta v_{k, \mathrm{RFS}} \times 10^{-m}$ with $m=3$, which has been used so far for determining the assumed uncertainty in the $\delta v_{k, \text { RFS }}$ values.

The extracted radial moments together with the resulting errors are displayed in Table VIII. Comparing the respective results of Table IV with the results in Table VIII, we deduce that although in the latter case the systematical errors are larger the statistical errors of the extracted $\delta\left\langle r^{2}\right\rangle$ and $\delta\left\langle r^{4}\right\rangle$ values are significantly smaller. We see that now the relative statistical errors are $\frac{\Delta\left(\delta\left\langle r^{2}\right\rangle\right)}{\left|\delta\left\langle r^{2}\right\rangle\right|}=0.022$ and $\frac{\Delta\left(\delta\left\langle r^{4}\right\rangle\right)}{\left|\delta\left\langle r^{4}\right\rangle\right|}=0.24$, respectively.

In Figs. 11 and 12, the relative errors in the extraction of the $\delta\left\langle r^{2}\right\rangle$ and $\delta\left\langle r^{4}\right\rangle$ moments are illustrated as a function of the $m$ value. As seen, the results are rather sensitive to the $m$ value and the relative error increases dramatically as the precision of the field shift values decreases. This is even more pronounced for the errors in the extracted $\delta\left\langle r^{4}\right\rangle$ moments. Nevertheless, for $m=3$ both $\delta\left\langle r^{2}\right\rangle$ and $\delta\left\langle r^{4}\right\rangle$ moments are extracted with satisfactory accuracy. Thus, we deduce that provided the current experimental precision in the isotope shift measurements, an accurate enough extraction of the $\delta\left\langle r^{2}\right\rangle$ and $\delta\left\langle r^{4}\right\rangle$ moments could be possible as long as the measured transitions are sufficiently independent in terms of electronic factors.

\section{SUMMARY AND CONCLUSIONS}

Combining nuclear DFT-type models with MCHF calculations for atomic states it is possible to achieve a higher precision in the predictions of atomic line field shifts. Changes in the nuclear charge distribution caused by shell structure, deformations, and variations in the diffuseness of the nuclei 


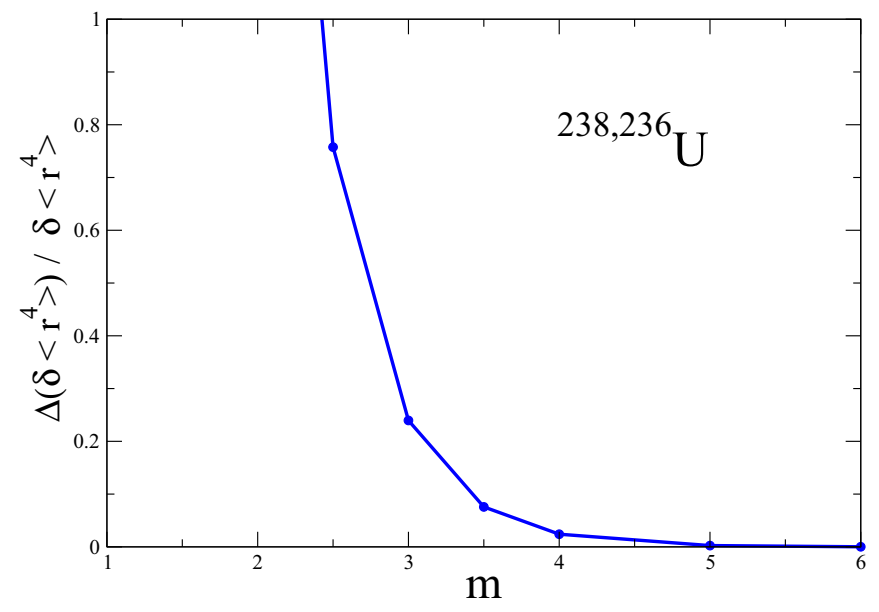

FIG. 12. Same as Fig. 11, but for the relative uncertainty in the extraction of the $\delta\left\langle r^{4}\right\rangle$ moment using the same pair of transitions.

are then automatically taken into account. In this work, it is shown that capturing all these effects leads to an improved description of experiments.

With the continuous advancement in experimental methods, one may ask whether the improved precision and access to several atomic transitions makes it possible to obtain more data on the nuclear isotopes than just the $\delta\left\langle r^{2}\right\rangle$ values commonly extracted so far. By constructing a set of theoretical field shifts we explore the possibility of extracting information about the nucleus by inverting the first-order perturbation theory equations for the field shifts. In this way, we demonstrate that the electron states are sensitive not only to the $\delta\left\langle r^{2}\right\rangle$ values but also to changes in $\left\langle r^{4}\right\rangle$ values. This opens the possibility for systematic tabulation of these higher-order nuclear moments. Considering both statistical and systematical errors in the extraction procedure we conclude that an increase in experimental precision by one to two orders of magnitude or access to data for more independent atomic transitions would be essential. As a promising candidate for future experiments, we suggest Li-like uranium where an increase in precision with one order of magnitude along with access to at least two independent transitions would allow accurate $\delta\left\langle r^{4}\right\rangle$ values to be extracted.

\section{ACKNOWLEDGMENTS}

This work was supported by the Swedish Research Council (VR). The authors acknowledge P. Jönsson, Malmö University, and C. Brandau, GSI, for discussions and C. Nazé, J. G. Li, and M. Godefroid for providing the barium MCDHF wave functions.

\section{APPENDIX: RFS EXPANSION IN ORTHONORMAL BASIS}

The RFS is, for a certain transition, given by the expansion

$$
\sum_{N=1}^{4} F_{N} \delta\left\langle r^{2 N}\right\rangle=F_{1} \delta\left\langle r^{2}\right\rangle+F_{2} \delta\left\langle r^{4}\right\rangle+F_{3} \delta\left\langle r^{6}\right\rangle+F_{4} \delta\left\langle r^{8}\right\rangle
$$

where the line field shift factors $F_{N}$ play the role of expansion coefficients. The set of $r^{2 N}$ that forms the basis $\left\{r^{2}, r^{4}, r^{6}, r^{8}\right\}$ is not orthonormal. It is reasonable to assume that a rearrangement using an orthonormal basis should lead to faster convergence. Here, we orthonormalize the initial basis with respect to the scalar product:

$$
\langle u \mid v\rangle=\int u * v * w r^{2} d r,
$$

where $w$ is the weight function that approximates the nucleus. Since the functions $y_{N}$, forming the basis $\left\{y_{1}, y_{2}, y_{3}, y_{4}\right\}$, are constructed to be orthogonal they will probe different aspects of the nuclear charge distribution within the nuclear volume. Thus, we expect that the expansion

$$
\sum_{N=1}^{4} c_{N} \delta\left\langle y_{N}\right\rangle=c_{1} \delta\left\langle y_{1}\right\rangle+c_{2} \delta\left\langle y_{2}\right\rangle+c_{3} \delta\left\langle y_{3}\right\rangle+c_{4} \delta\left\langle y_{4}\right\rangle
$$

will converge faster than $\sum_{N=1}^{4} F_{N} \delta\left\langle r^{2 N}\right\rangle$ does. In the expression above, $c_{N}$ are the expansion coefficients. Assuming that the nucleus can be approximated as a hard sphere, one can use $w=\rho_{0} \Theta(R-r)$ with $R=1.25 A^{1 / 3}$. The value of $\rho_{0}$ is determined by the normalization condition $4 \pi \int \rho_{0} r^{2} d r=$ 1. Following the Gram-Schmidt process [44], we obtain

$$
\begin{aligned}
& y_{1}=\frac{3.46556}{\bar{A}^{2 / 3}} r^{2}, \\
& y_{2}=-\frac{15.2051}{\bar{A}^{2 / 3}} r^{2}+\frac{12.5116}{\bar{A}^{4 / 3}} r^{4}, \\
& y_{3}=\frac{39.9503}{\bar{A}^{2 / 3}} r^{2}-\frac{80.3573}{\bar{A}^{4 / 3}} r^{4}+\frac{37.1429}{\bar{A}^{2}} r^{6}, \\
& y_{4}=-\frac{82.4315}{\bar{A}^{2 / 3}} r^{2}+\frac{293.927}{\bar{A}^{4 / 3}} r^{4}-\frac{313.522}{\bar{A}^{2}} r^{6}+\frac{103.367}{\bar{A}^{8 / 3}} r^{8},
\end{aligned}
$$

where $\bar{A}$ is taken as the average of the mass numbers of the two isotopes. The sum of the expansion terms has been rearranged but $\sum_{N=1}^{4} F_{N} \delta\left\langle r^{2 N}\right\rangle=\sum_{N=1}^{4} c_{N} \delta\left\langle y_{N}\right\rangle$ must still hold. The $c_{N}$ coefficients can be found by equating same order terms in the above equation. Hence, the new coefficients are

$$
\begin{aligned}
c_{1}= & 0.288554 \bar{A}^{2 / 3} F_{1}+0.350673 \bar{A}^{4 / 3} F_{1} \\
& +0.448303 \bar{A}^{2} F_{3}+0.592709 \bar{A}^{8 / 3} F_{4}, \\
c_{2}= & 0.0799258 \bar{A}^{4 / 3} F_{2}+0.172916 \bar{A}^{2} F_{3}+0.2972 \bar{A}^{8 / 3} F_{4}, \\
c_{3}= & 0.026923 \bar{A}^{2} F_{3}+0.08166 \bar{A}^{8 / 3} F_{4}, \\
c_{4}= & 0.00967424 \bar{A}^{8 / 3} F_{4} .
\end{aligned}
$$

Now, the RFS is given by the summation

$$
\sum_{N=1}^{4} c_{N} \delta\left\langle y_{N}\right\rangle
$$

and the matching percentage to the final field shift after each term has been added differs from the one when the original summation is used.

As seen in Fig. 9, the orthogonal expansion converges substantially faster than the original summation. In fact, only the $\delta\left\langle r^{2}\right\rangle$ and $\delta\left\langle r^{4}\right\rangle$ moments need to be considered as long as the sum is rearranged. Thus, for a pair of isotopes $A, A^{\prime}$ 
and a transition $k$, the RFS is to a very good approximation expressed as

$$
\delta v_{k, \mathrm{RFS}}^{A, A^{\prime}} \approx c_{k, 1} \delta\left\langle y_{1}\right\rangle+c_{k, 2} \delta\left\langle y_{2}\right\rangle .
$$

In case the isotope shifts are known for two transitions, a system of two equations can be formed, and the $c_{k, 1}$ and $c_{k, 2}$ constants can be evaluated using the expressions above. They depend on the line field shift factors $F_{k, N}$ that are different for each transition and which are calculated for the reference isotope $A$. Therefore, for two transitions, the problem takes the form of a matrix equation

$$
\left[\begin{array}{l}
\delta v_{1, \mathrm{RFS}}^{A, A^{\prime}} \\
\delta v_{2, \mathrm{RFS}}^{A, A^{\prime}}
\end{array}\right] \approx\left[\begin{array}{ll}
c_{1,1} & c_{1,2} \\
c_{2,1} & c_{2,2}
\end{array}\right]\left[\begin{array}{l}
\delta\left\langle y_{1}\right\rangle \\
\delta\left\langle y_{2}\right\rangle
\end{array}\right]
$$

The unknown $y_{1}$ and $y_{2}$ can thus be solved according to

$$
\left[\begin{array}{l}
\delta\left\langle y_{1}\right\rangle \\
\delta\left\langle y_{2}\right\rangle
\end{array}\right] \approx C^{-1}\left[\begin{array}{l}
\delta v_{1, \mathrm{RFS}}^{A, A^{\prime}} \\
\delta v_{2, \mathrm{RFS}}^{A, A^{\prime}}
\end{array}\right]
$$

where $C^{-1}$ is the inverse matrix of $\left[\begin{array}{ll}c_{1,1} & c_{1,2} \\ c_{2,1} & c_{2,2}\end{array}\right]$. The $\delta\left\langle r^{2}\right\rangle$ and $\delta\left\langle r^{4}\right\rangle$ moments are finally extracted by solving the equations

$$
\left[\begin{array}{l}
\delta\left\langle y_{1}\right\rangle \\
\delta\left\langle y_{2}\right\rangle
\end{array}\right]=\left[\begin{array}{cc}
3.46556 / \bar{A}^{2 / 3} & 0 \\
-15.2051 / \bar{A}^{2 / 3} & 12.5116 / \bar{A}^{4 / 3}
\end{array}\right]\left[\begin{array}{l}
\delta\left\langle r^{2}\right\rangle \\
\delta\left\langle r^{4}\right\rangle
\end{array}\right] .
$$

This can be compared with the original summation, where if the approximate relation

$$
\delta v_{k, \mathrm{RFS}}^{A, A^{\prime}} \approx F_{k, 1} \delta\left\langle r^{2}\right\rangle+F_{k, 2} \delta\left\langle r^{4}\right\rangle
$$

is assumed, the matrix equation to be solved is given by

$$
\left[\begin{array}{l}
\delta v_{1, \mathrm{RFS}}^{A, A^{\prime}} \\
\delta v_{2, \mathrm{RFS}}^{A, A^{\prime}}
\end{array}\right] \approx\left[\begin{array}{ll}
F_{1,1} & F_{1,2} \\
F_{2,1} & F_{2,2}
\end{array}\right]\left[\begin{array}{l}
\delta\left\langle r^{2}\right\rangle \\
\delta\left\langle r^{4}\right\rangle
\end{array}\right]
$$

[1] I. Angeli and K. Marinova, Table of experimental nuclear ground state charge radii: An update, At. Data Nucl. Data Tables 99, 69 (2013).

[2] C. G. Parthey et al., Precision measurement of the hydrogendeuterium $1 s-2 s$ isotope shift, Phys. Rev. Lett. 104, 233001 (2010).

[3] S. E. Agbemava, A. V. Afanasjev, D. Ray, and P. Ring, Global performance of covariant energy density functionals: Ground state observables of even-even nuclei and the estimate of theoretical uncertainties, Phys. Rev. C 89, 054320 (2014).

[4] D. Andrae, Finite nuclear charge density distributions in electronic structure calculations for atoms and molecules, Phys. Rep. 336, 413 (2000).

[5] Y. S. Kozhedub, O. V. Andreev, V. M. Shabaev, I. I. Tupitsyn, C. Brandau, C. Kozhuharov, G. Plunien, and T. Stöhlker, Nuclear deformation effect on the binding energies in heavy ions, Phys. Rev. A 77, 032501 (2008).

[6] N. A. Zubova, Y. S. Kozhedub, V. M. Shabaev, I. I. Tupitsyn, A. V. Volotka, G. Plunien, C. Brandau, and Th. Stöhlker, Relativistic calculations of the isotope shifts in highly charged Li-like ions, Phys. Rev. A 90, 062512 (2014).

[7] M. J. G. Borge, Recent highlights of the isolde facility, J. Phys.: Conf. Ser. 580, 012049 (2015).

[8] C. Brandau, M. Lestinsky, C. Kozhuharov, A. Müller, S. Schippers, and Th. Stöhlker, High-resolution dielectronic recombination experiments at the upcoming cryring@esr facility, J. Phys.: Conf. Ser. 635, 022084 (2015).

[9] C. W. P. Palmer, Reformulation of the theory of the mass shift, J. Phys. B: At. Mol. Phys. 20, 5987 (1987).

[10] V. M. Shabaev, Mass corrections in a strong nuclear field, Theor. Math. Phys. 63, 588 (1985).

[11] V. Shabaev and A. Artemyev, Relativistic nuclear recoil corrections to the energy levels of multicharged ions, J. Phys. B: At. Mol. Phys. 27, 1307 (1994).

[12] E. C. Seltzer, $k$ x-ray isotope shifts, Phys. Rev. 188, 1916 (1969).

[13] G. Torbohm, B. Fricke, and A. Rosén, State-dependent volume isotope shifts of low-lying states of group-ii $a$ and -ii $b$ elements, Phys. Rev. A 31, 2038 (1985).
[14] S. Blundell, P. Baird, C. Palmer, D. Stacey, and G. Woodgate, A reformulation of the theory of field isotope shift in atoms, J. Phys. B: At. Mol. Phys. 20, 3663 (1987).

[15] J. Ekman et al. [Comp. Phys. Comm. (unpublished)]

[16] P. Jönsson, G. Gaigalas, J. Bieroń, C. Froese Fischer, and I. Grant, New version: Grasp2k relativistic atomic structure package, Comput. Phys. Commun. 184, 2197 (2013).

[17] I. P. Grant, Springer Series on Atomic, Optical and Plasma Physics, Vol. 40 (Springer, Berlin, 2007).

[18] B. J. McKenzie, I. P. Grant, and P. H. Norrington, A program to calculate transverse breit and qed corrections to energy levels in a multiconfiguration dirac-fock environment, Comput. Phys. Commun. 21, 233 (1980).

[19] C. Nazé, E. Gaidamauskas, G. Gaigalas, M. Godefroid, and P. Jönsson, ris3: A program for relativistic isotope shift calculations, Comput. Phys. Commun. 184, 2187 (2013).

[20] S. A. Blundell, W. R. Johnson, and J. Sapirstein, Improved many-body perturbation theory calculations of the $n=2$ states of lithiumlike uranium, Phys. Rev. A 41, 1698 (1990).

[21] W. R. Johnson, J. Sapirstein, and K. T. Cheng, Theory of $2 s_{1 / 2}-2 p_{3 / 2}$ transitions in highly ionized uranium, Phys. Rev. A 51, 297 (1995).

[22] J. D. Zumbro, E. B. Shera, Y. Tanaka, C. E. Bemis, R. A. Naumann, M. V. Hoehn, W. Reuter, and R. M. Steffen, $e 2$ and $e 4$ deformations in ${ }^{233,234,235,238}$ U, Phys. Rev. Lett. 53, 1888 (1984).

[23] J. D. Zumbro, R. A. Naumann, M. V. Hoehn, W. Reuter, E. B. Shera, C. E. Bemis, and Y. Tanaka, E2 and e4 deformations in ${ }^{232}$ Th and ${ }^{239,240,242} \mathrm{Pu}$, Phys. Lett. B 167, 383 (1986).

[24] P. Ring and P. Schuck, The Nuclear Many-Body Problem, 1st ed. (Springer, New York, 1980).

[25] E. Chabanat, P. Bonche, P. Haensel, J. Meyer, and R. Schaeffer, A Skyrme parametrization from subnuclear to neutron star densities Part ii. Nuclei far from stabilities, Nucl. Phys. A 635, 231 (1998).

[26] B. A. Brown, New Skyrme interaction for normal and exotic nuclei, Phys. Rev. C 58, 220 (1998).

[27] B. G. Carlsson, J. Dobaczewski, J. Toivanen, and P. Veselý, Solution of self-consistent equations for the $\mathrm{N}^{3} \mathrm{LO}$ nuclear 
energy density functional in spherical symmetry. The program hosphe (v1.02), Comput. Phys. Commun. 181, 1641 (2010).

[28] M. Stoitsov, N. Schunck, M. Kortelainen, N. Michel, H. Nam et al., Axially deformed solution of the Skyrme-Hartree-FockBogoliubov equations using the transformed harmonic oscillator basis (ii) HFBTHO v2.00d: A new version of the program, Comput. Phys. Commun. 184, 1592 (2013).

[29] A. Bouyssy, J. F. Mathiot, N. Van Giai, and S. Marcos, Relativistic description of nuclear systems in the Hartree-Fock approximation, Phys. Rev. C 36, 380 (1987).

[30] R. Rosenfelder, Coulomb corrections to elastic electronproton scattering and the proton charge radius, Phys. Lett. B 479, 381 (2000).

[31] R. Pohl, R. Gilman, G. Miller, and K. Pachucki, Muonic Hydrogen and the Proton Radius Puzzle, Annu. Rev. Nucl. Part. Sci. 63, 175 (2013).

[32] H. De Vries, C. W. De Jager, and C. De Vries, Nuclear ChargeDensity-Distribution Parameters from Electron Scattering, At. Data Nucl. Data Tables 36, 495 (1987).

[33] G. Fricke, C. Bernhardt, K. Heilig, L. A. Schaller, L. Schellenberg, E. B. Shera, and C. W. Dejager, Nuclear ground state charge radii from electromagnetic interactions, At. Data Nucl. Data Tables 60, 177 (1995).

[34] W. van Wijngaarden and J. Li, Hyperfine splittings and isotope shifts of (6s)21s0-(6s6p) 1p1 transition in barium, Can. J. Phys. 73, 484 (1995).

[35] C. Nazé, J. G. Li, and M. Godefroid, Theoretical isotope shifts in neutral barium, Phys. Rev. A 91, 032511 (2015).

[36] J. Li, C. Nazé, M. Godefroid, S. Fritzsche, G. Gaigalas, P. Indelicato, and P. Jönsson, Mass- and field-shift isotope parameters for the $2 s-2 p$ resonance doublet of lithiumlike ions, Phys. Rev. A 86, 022518 (2012).

[37] C. Brandau, C. Kozhuharov, Z. Harman, A. Müller, S. Schippers, Y. S. Kozhedub, D. Bernhardt, S. Böhm, J. Jacobi, E. W. Schmidt, P. H. Mokler, F. Bosch, H.-J. Kluge, Th. Stöhlker, K. Beckert, P. Beller, F. Nolden, M. Steck, A. Gumberidze, R. Reuschl, U. Spillmann, F. J. Currell, I. I. Tupitsyn, U. D. Jentschura, V. M. Shabaev, C. H. Keitel, A. Wolf, and Z Stachura, Isotope Shift in the Dielectronic Recombination of Three- Electron ${ }^{A} \mathrm{Nd}^{57+}$, Phys. Rev. Lett. 100, 073201 (2008).

[38] S. R. Elliott, P. Beiersdorfer, and M. H. Chen, Trapped-Ion Technique for Measuring the Nuclear Charge radii of Highly Charged Radioactive Isotopes, Phys. Rev. Lett. 76, 1031 (1996).

[39] G. Plunien, B. Müller, W. Greiner, and G. Soff, Nuclear polarization contribution to the lamb shift in heavy atoms, Phys. Rev. A 39, 5428 (1989).

[40] G. Plunien, B. Müller, W. Greiner, and G. Soff, Nuclear polarization in heavy atoms and superheavy quasiatoms, Phys. Rev. A 43, 5853 (1991).

[41] G. Plunien and G. Soff, Nuclear-polarization contribution to the lamb shift in actinide nuclei, Phys. Rev. A 51, 1119 (1995).

[42] G. Plunien and G. Soff, Erratum: Nuclear-polarization contribution to the lamb shift in actinide nuclei, Phys. Rev. A 53, 4614 (1996).

[43] T. Beier, P. J. Mohr, H. Persson, and G. Soff, Influence of nuclear size on QED corrections in hydrogenlike heavy ions, Phys. Rev. A 58, 954 (1998).

[44] E. W. Weisstein, Gram-Schmidt orthonormalization, from MATHWORLD, a Wolfram web resource. 